ARTICLE

DOI: $10.1038 / s 41467-018-07009-z$

\title{
Onsite GTP fuelling via DYNAMO1 drives division of mitochondria and peroxisomes
}

Yuuta Imoto ${ }^{1}$, Yuichi Abe ${ }^{1}$, Masanori Honsho', Kanji Okumoto ${ }^{2}$, Mio Ohnuma ${ }^{3}$, Haruko Kuroiwa ${ }^{4}$, Tsuneyoshi Kuroiwa ${ }^{4}$ \& Yukio Fujiki [D ${ }^{1}$

Mitochondria and peroxisomes proliferate by division. During division, a part of their membrane is pinched off by constriction of the ring-shaped mitochondrial division (MD) and peroxisome-dividing (POD) machinery. This constriction is mediated by a dynamin-like GTPase Dnm1 that requires a large amount of GTP as an energy source. Here, via proteomics of the isolated division machinery, we show that the 17-kDa nucleoside diphosphate kinaselike protein, dynamin-based ring motive-force organizer 1 (DYNAMO1), locally generates GTP in MD and POD machineries. DYNAMO1 is widely conserved among eukaryotes and colocalizes with Dnm1 on the division machineries. DYNAMO1 converts ATP to GTP, and disruption of its activity impairs mitochondrial and peroxisomal fissions. DYNAMO1 forms a ring-shaped complex with Dnm1 and increases the magnitude of the constricting force. Our results identify DYNAMO1 as an essential component of MD and POD machineries, suggesting that local GTP generation in Dnm1-based machinery regulates motive force for membrane severance.

\footnotetext{
${ }^{1}$ Division of Organelle Homeostasis, Medical Institute of Bioregulation, Kyushu University, 3-1-1 Maidashi, Higashi-ku, Fukuoka 812-8582, Japan.

${ }^{2}$ Department of Biology, Faculty of Sciences, Kyushu University, 744 Motooka Nishi-ku, Fukuoka 819-0395, Japan. ${ }^{3}$ Institute of Technology, Hiroshima College, 4272-1 Higashino, Osaki kamijima-cho, Toyota-gun, Hiroshima 725-0231, Japan. ${ }^{4}$ Department of Chemical and Biological Science, Faculty of Science, Japan Women's University, 2-8-1 Mejirodai, Bunkyo-ku, Tokyo 112-8681, Japan. Correspondence and requests for materials should be addressed to Y.F. (email: yfujiki@kyudai.jp)
} 
M embrane fission is essential for the life of cells. During cell division, the cell membrane is cleaved at the equatorial plane. Cellular communication from hormone signaling to neurotransmission is supported by a process known as endocytosis. For proliferation of intracellular organelles such as mitochondria and peroxisomes, portions of their membrane are severed to generate daughter organelles. These fission reactions are essential for all intracellular membrane remodeling events and are mediated by the dynamin family of GTPase proteins ${ }^{1}$. These proteins polymerize and form a ring or spiral structure to constrict and pinch off the membrane ${ }^{2}$. Typical dynamin family members are Dnm1, which mediates division of mitochondria ${ }^{3}$ and peroxisomes ${ }^{4}$, and dynamin isoforms that pinch off the neck of endocytic pits ${ }^{5}$. They are among the most powerful motor proteins and are capable of constricting membrane gaps as large as a few hundred nanometers in less than $1 \min ^{6,7}$. Because the diameters of mitochondrial and peroxisomal division planes are substantially larger than the neck of an endocytic pit, the magnitude of the constriction of in vitro Dnm1 $(\sim 50 \mathrm{~nm})$ is fivefold higher than that of dynamin $(\sim 10 \mathrm{~nm})^{7}$. Despite the high magnitude of its constriction, Dnm1 has a weak affinity for GTP and relatively high rate of GTP hydrolysis ${ }^{8}$ as similar to dynamin ${ }^{9}$. Furthermore, high rate of GTPase activity is enhanced by polymerization $\left(k_{\text {cat }}=50 \mathrm{~min}^{-1}\right)^{8}$ much higher than that of small GTPase $\left(k_{\text {cat }}=\sim 8 \times 10^{-3} \mathrm{~min}^{-1}\right)^{10}$. The fact that significant constriction mediated by Dnml requires high levels of GTP $(\sim 1 \mathrm{mM})^{7}$, indicates that efficient replenishment of GTP is needed to sustain the activity of assembly and constriction. However, how the functions of Dnm1 are energetically supported is unclear. Accessory proteins of Dnm1, such as Fis1, Mdv1, Mff, and MiDs, might enhance the kinetics of its GTP affinity and facilitate membrane constriction ${ }^{11}$. However, conservation of these proteins among eukaryotes is limited, even though Dnm1-mediated division of mitochondria and peroxisomes is common to almost all eukaryotic cells including those in animals, fungi, land plants, and algae ${ }^{12}$.

To search for a fundamental protein that potentially functions in energetic regulation and kinetics of Dnm1, proteomic analysis of the division machinery is required. For this approach, isolation of the division machineries is essential. However, most of the eukaryotic cells contain numerous numbers of mitochondria and peroxisomes that divide randomly, which makes it difficult to collect bulk of intermediate of dividing organelles. In contrast, a unicellular red alga, Cyanidioschyzon merolae (C. merolae), has advantages to conquer this problem because it contains only one peroxisome, mitochondrion, and plastid per cell. Moreover, division of these organelles can be highly synchronized by light/dark stimulation in C. merolae (Supplementary Figure 1) ${ }^{13,14}$. This feature enables bulk isolation of mitochondrial and peroxisomal division machineries ${ }^{15-17}$. The division machinery of mitochondria, called mitochondrial division (MD) machinery, has a ring-shaped electron-dense structure with a diameter of $150-1200 \mathrm{~nm}^{12}$. This machinery consists of an outer ring formed around the cytoplasmic side of the mitochondrial outer membrane and an inner ring formed around the matrix side of the inner membrane. The outer ring is a dynamin-based ring containing Dnm1 and skeletal polyglucan filaments, called the MD ring ${ }^{16,18,19}$. The inner ring is a remnant of a bacterial cell division apparatus containing Fts ${ }^{20}$ that Opisthokonta have $\operatorname{lost}^{21,22}$. However, MD machinery is physically associated with plastid dividing $(\mathrm{PD})$ machinery, implying that the isolated MD machinery fraction may be contaminated with plastid division-associated proteins ${ }^{15,16,23}$. In contrast, the division machinery of peroxisomes is formed at different times and sites than MD and PD machineries ${ }^{17,24}$. The peroxisome-dividing
(POD) machinery with a diameter of $50-600 \mathrm{~nm}$ is composed of dynamin-based (DB) rings and skeletal filamentous rings formed at the cytoplasmic side of peroxisomal membranes ${ }^{17}$. The DB ring is structurally analogous to the outer ring of MD machinery and also contains Dnm $1{ }^{17}$. Moreover, the DB ring can be physically separated from the other components of $\mathrm{POD}$ machinery, which enables mapping of candidate proteins to specific structures.

By using the unicellular red alga, C. merolae, $17-\mathrm{kDa}$ nucleoside diphosphate kinase-like protein, dynamin-based ring motiveforce organizer 1 (DYNAMO1) is identified as a fundamental component of MD and POD machineries. DYNAMO1 directly binds to Dnm1 and localizes on the Dnm1-based ring structure in the machineries. DYNAMO1 generates GTP from ATP and GDP and this enzyme activity is essential to sustain Dnm1-mediated fission of mitochondrion and peroxisome. Furthermore, in vitro analysis demonstrates that DYNAMO1 enhances constriction force generated by Dnm1-based rings. Thus, energy source for the constriction of $\mathrm{MD}$ and $\mathrm{POD}$ machineries is most likely generated locally around the membrane scission sites during the division of mitochondrion and peroxisome.

\section{Results}

Identification of DYNAMO1 from isolated POD machinery. We investigated the molecular identity of Dnml-based machinery by proteomic analysis of the POD machinery-enriched fraction (Fig. 1a, b). To prepare sufficient amounts of POD machinery, we improved existing isolation methods, including treatment with $0.2 \%$ lauryldimethylamine-N-oxide (LDAO) instead of $0.8 \% n$-octyl- $\beta$-D-glucoside $(\mathrm{OG})^{17,25}$, to greatly increase the yield of Dnm1-positive rings (Fig. 1c, d; Supplementary Figure 2a and b). The LDAO-treated fraction of POD machinery contained spiral structures (Fig. 2d) similar to those formed by Dnml GTPase in MD machinery ${ }^{15}$. Thus, the LDAOtreated fraction of POD machinery possibly contained intact POD machinery with retention of its in vivo functions. To identify components of the DB ring, the solubilized fraction of isolated POD machinery was compared with the interphase fraction. Six bands distinctly enriched in the POD machinery fraction were analyzed by liquid chromatography and mass spectrometry (LC-MS/MS) and peptide mass fingerprinting using the complete genomic sequence of C. merolae ${ }^{14,26}$. Dnm1 was evidently identified in band 1 at $\sim 90 \mathrm{kDa}$ (Supplementary Figure 2c; Supplementary Table 1), indicating enrichment of POD machinery. Among the detected candidate proteins, we found that the gene product of $C M L 110 C$, a top-assigned protein in the most enriched band 5 (Supplementary Figure 2c; Supplementary Table 1), bound to Dnm1 in vitro and in vivo (Supplementary Figure $2 \mathrm{~d}-\mathrm{g}$ ), suggesting that CML110C is a novel $17 \mathrm{kDa}$ component of the $\mathrm{DB}$ ring. CML110C, termed as DYNAMO1, contains a consensus motif of nucleoside diphosphate kinase (NDPK) (Supplementary Figure 2h) that forms various oligomeric structures, such as a hexamer, tetramer, and dimer, and catalyzes conversion of ATP to GTP $27-29$. Recombinant DYNAMO1 formed a tetramer (Supplementary Figure 2i-k) and generated GTP upon addition of ATP plus GDP (Fig. 1e, f). When the histidine residue at position 116 of DYNAMO1 in the consensus active site motif was replaced by aspartic acid (DYNAMO1 H116D), its GTP generation was abolished (Fig. 1e), demonstrating that DYNAMO1 indeed functions as a nucleoside diphosphate kinase. Because nucleoside diphosphate kinase is involved in dynamin-mediated endocytosis and OPA1-mediated mitochondrial fusion in mammalian cells ${ }^{29,30}$, we suggest that DYNAMO1 is involved in Dnm1-based organelle division machineries. 
a

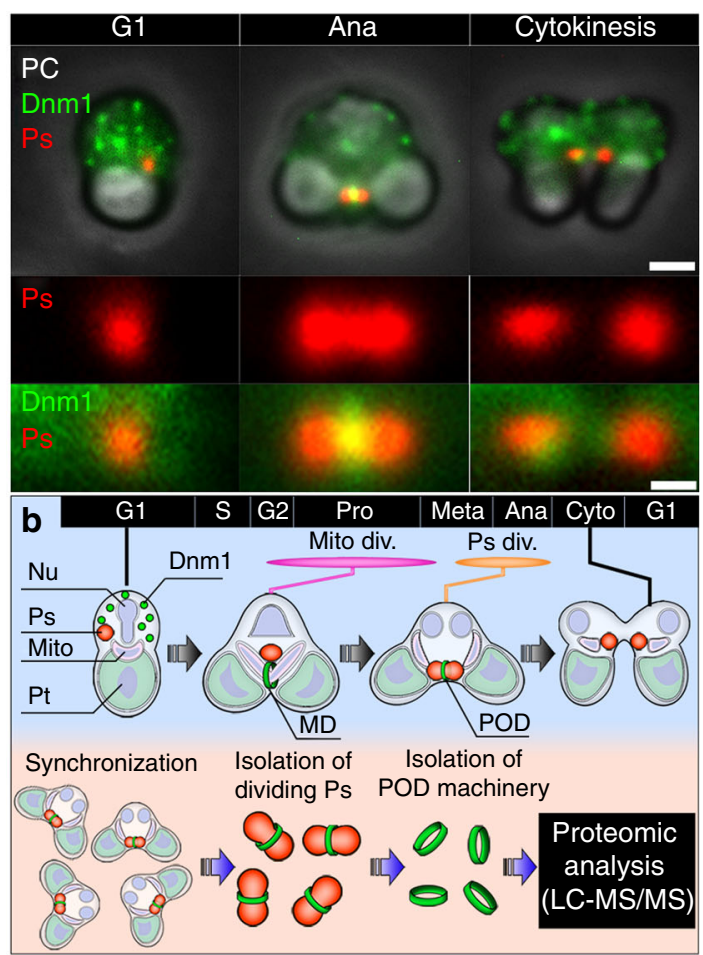

C
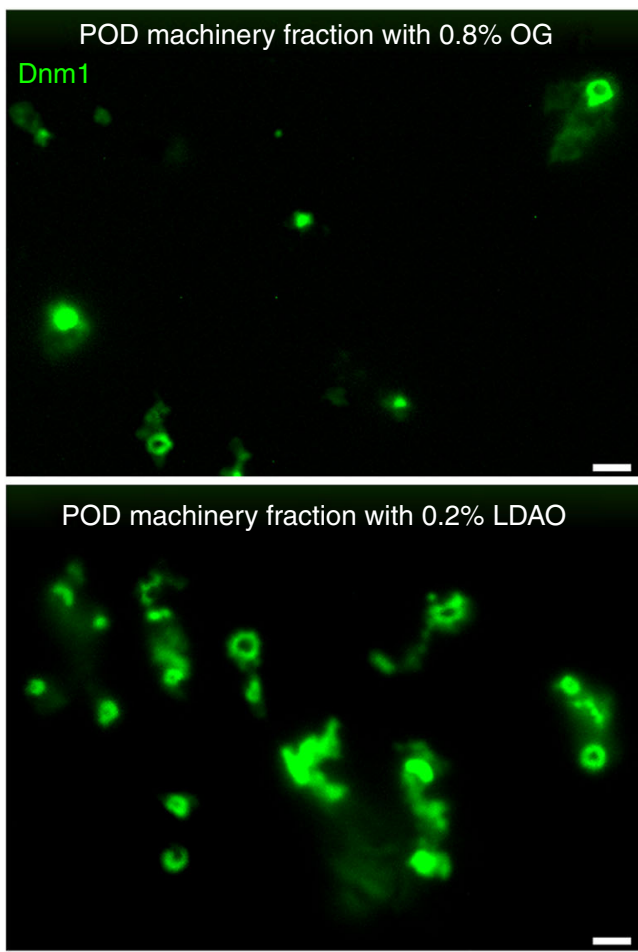

$\mathbf{f}$ d

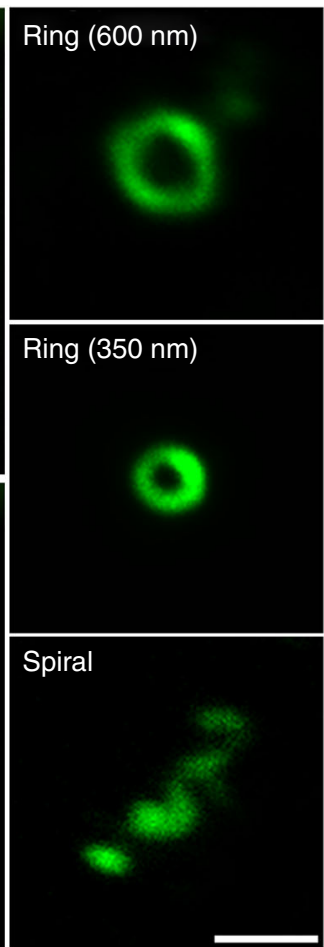

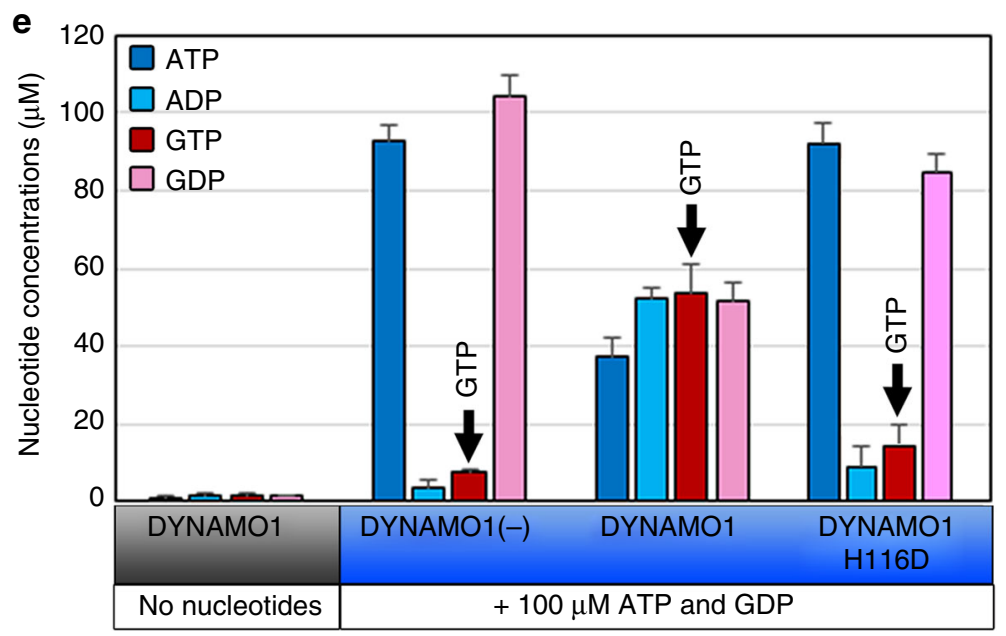
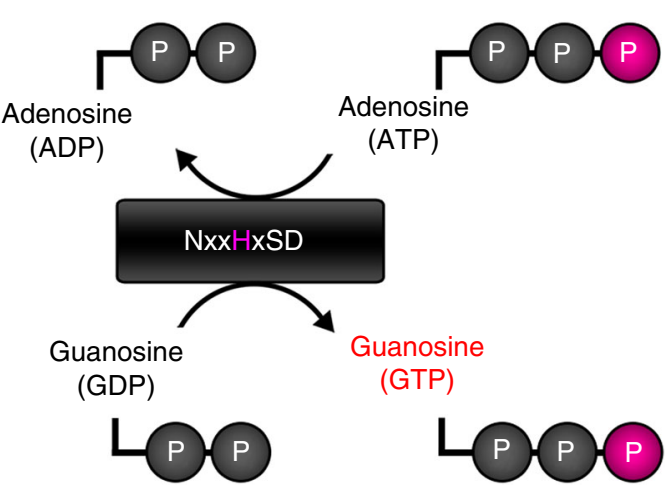

Fig. 1 Proteomic analysis of POD machinery and identification of DYNAMO1. a Phase contrast (PC) and immunofluorescence images of C. merolae cells during G1 phase, anaphase, and cytokinesis. Ps peroxisome (anti-catalase antibody), Dnm1 (anti-Dnm1 antibody). b Schematic representing isolation and proteomic analysis of POD machinery. Nu cell nucleus, Mito mitochondrion, Pt plastid, Mito div. mitochondrial division period, Ps div peroxisomal division period. c Upper panel shows the 0.8\% OG-treated POD machinery fraction and the lower panel shows the 0.2\% LDAO-treated POD machinery fraction. $\mathbf{d}$ Typical structures of isolated POD machinery stained with the anti-Dnm1 antibody. e LC-ESI-MS/MS analysis of the nucleoside diphosphate kinase activity of recombinant DYNAMO1. $\mathbf{f}$ Schematic representing a working model of nucleoside diphosphate kinase. Data in $\mathbf{e}$ are means \pm s.d. $(n=3)$. Scale bars: 1 $\mu \mathrm{m}$ (a, upper panels); $500 \mathrm{~nm}$ (a, lower panels; c, d)

Localization of DYNAMO1 at the organelle division sites. To investigate whether DYNAMO1 is involved in organelle division, we immunocytochemically analyzed endogenous DYNAMO1 with an anti-DYNAMO1 antibody. DYNAMO1 was expressed continuously during the cell cycle and was detected in both fractions of organelles containing peroxisomes and mitochondria and cytosol (Supplementary Figure 3a and b). Notably, 15-25\% of DYNAMO1 were detected in organelle fractions as compared to cytosolic fraction at the G2-M cell cycle phase, when mitochondria and peroxisomes divide (Supplementary Figure 3b-d). Therefore, DYNAMO1 is likely involved in not only peroxisomal, but also mitochondrial division. We next analyzed localization of DYNAMO1 and Dnm1 at each cell cycle phase (Fig. 2). DYNAMO1 was initially distributed in the cytosol during G1 phase and then partially accumulated at both edges of the mitochondrion during $\mathrm{S}$ phase when the inner FtsZ ring formed at the destined division site of each mitochondrion (Supplementary Figure $4 \mathrm{a}$ and b). Subsequently, DYNAMO1 was recruited to Dnm1-labeled division sites of mitochondria during G2-prophase (Fig. 2a, b). Immediately after mitochondrial division, puncta of DYNAMO1 and Dnm1 apparently disappeared from the mitochondria. Furthermore, DYNAMO1 was almost 
a
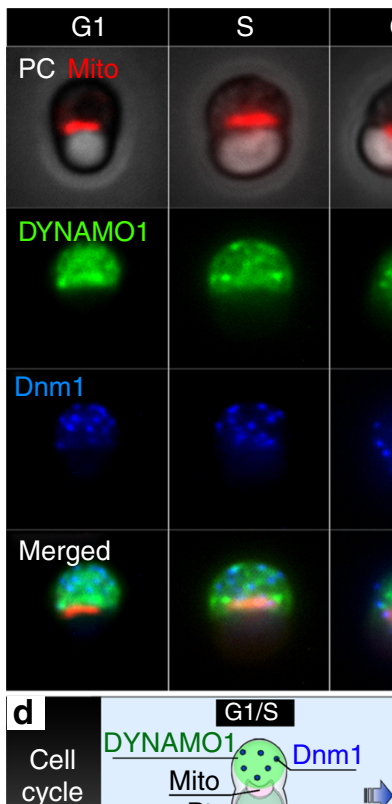

cycle

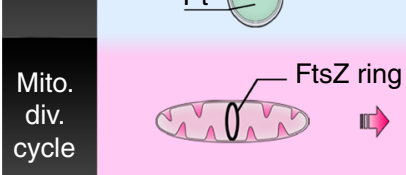

cycle

e

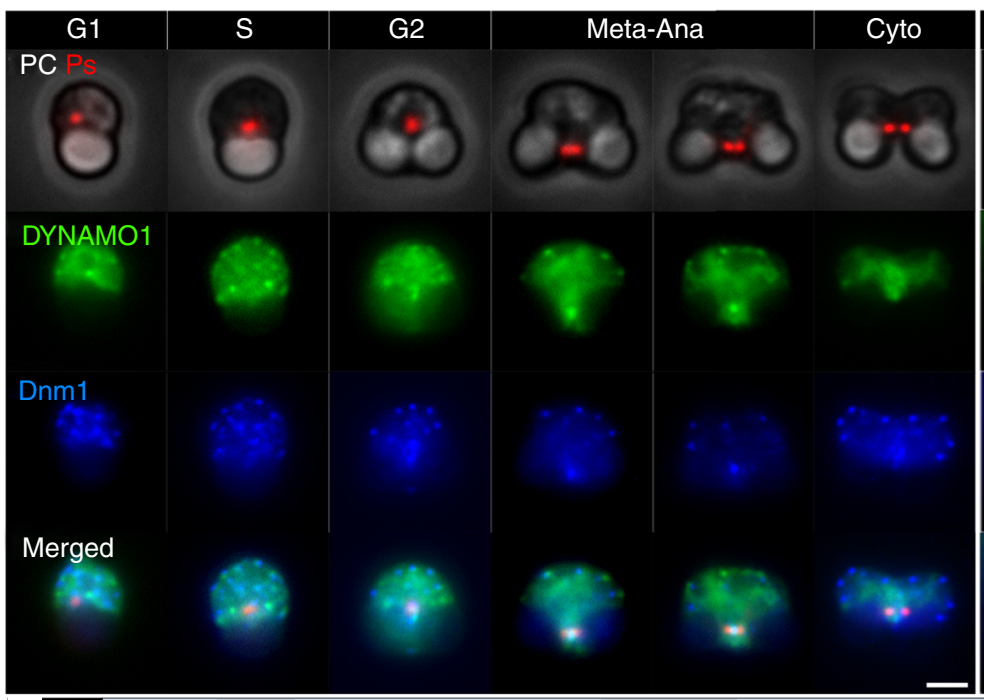

$\mathrm{h}$

Cell
cycle


Ps.
div.
cycle

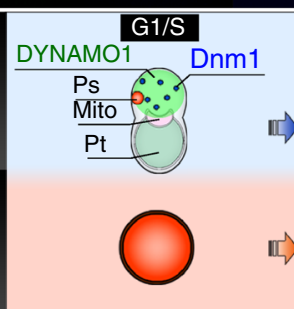

G2
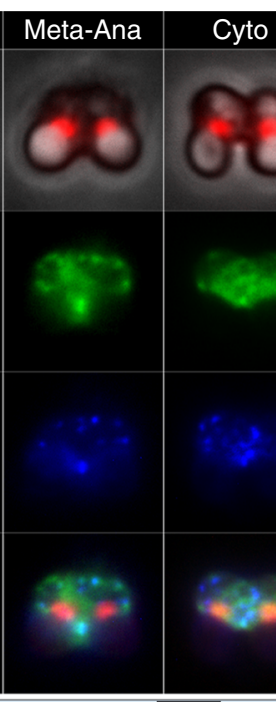

G2

Recruitment of
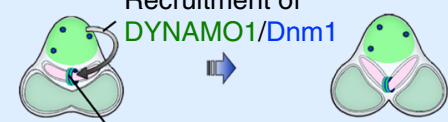

MD machinery with DYNAMO1/Dnm1

Mito

C

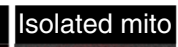

PC

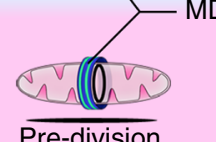

iil)

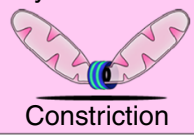

II)

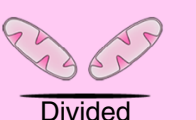

Divided

g

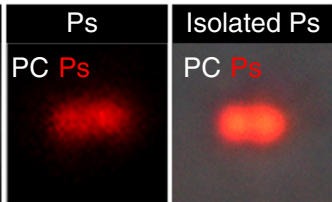

DYNAMO1

DYNAMO1
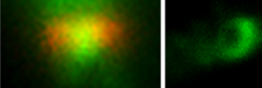

Dnm1 Dnm1

Merged Merged

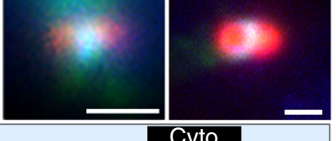

Recruitment of
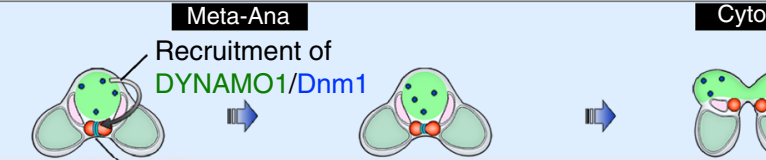

POD machinery with DYNAMO1/Dnm1

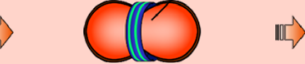

nd)

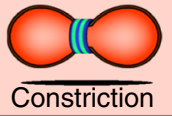

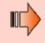

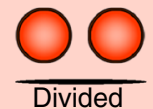

completely colocalized with Dnm1 at the division site and across the mitochondrial outer membrane of isolated mitochondria (Fig. 2c; Supplementary Figure 4c). Therefore, the dynamics of DYNAMO1 were cooperative with Dnm1 as a likely component of the outer ring of MD machinery (Fig. 2d). After mitochondrial division, DYNAMO1 was localized to the division sites of peroxisomes only during metaphase-anaphase and those of isolated peroxisomes (Fig. 2e-h; Supplementary Figure 4d). After 
Fig. 2 Dynamics of DYNAMO1 during the division periods of mitochondria and peroxisomes. a Phase contrast and immunofluorescence microscopy images of a mitochondrion (Mito, anti-EF-Tu antibody), DYNAMO1 (anti-DYNAMO1 antibody), and Dnm1 (anti-Dnm1 antibody) during the indicated cell cycle phases. Pro prophase, Meta-Ana metaphase-anaphase, Cyto cytokinesis. b Magnified image around the mitochondrial division site during prophase shown in a. c Localization of DYNAMO1 and Dnm1 on the isolated mitochondrial outer membrane [Mito-OM, anti-porin (POR) antibody]. d Schematic representing DYNAMO1 dynamics during the mitochondrial division period. e Phase contrast and immunofluorescence microscopy images of a peroxisome (Ps), DYNAMO1, and Dnm1 during the indicated cell cycle phases. $\mathbf{f}$ Magnified image around the peroxisomal division site during meta-anaphase shown in e. $\mathbf{g}$ Localization of DYNAMO1 and Dnm1 on an isolated dividing peroxisome. $\mathbf{h}$ Schematic representing DYNAMO1 dynamics during the peroxisomal division period. Scale bars: $1 \mu \mathrm{m}(\mathbf{a}, \mathbf{e}) ; 500 \mathrm{~nm}(\mathbf{b}, \mathbf{c}, \mathbf{f}$, and $\mathbf{g}$ )

peroxisome division, DYNAMO1 and Dnm1 signals were absent in peroxisomes, suggesting that DYNAMO1 is a component of POD machinery.

DYNAMO1 is a basic component of MD and POD machinery. To confirm that DYNAMO1 is a component of MD and POD machineries, we investigated its localization in preparations of the isolated machineries (Fig. 3a-c; Supplementary Figure 5a). DYNAMO1 was colocalized with Dnm1 in MD machinery labeled with Mdal and FtsZ, but not in PD machinery labeled with the PD ring component PDR1 (Fig. 3a). The DYNAMO1 and Dnml signal intensities in MD machinery were constant, regardless of the diameter of the ring (Supplementary Figure 5b), suggesting that DYNAMO1 is a fundamental component of MD machinery and functions together with Dnm1 during its entire constriction process. In the isolated POD machinery, DYNAMO1 was also colocalized with Dnm1, and the DYNAMO1 and Dnm1 signal intensities were constant, regardless of the diameter of the POD ring (Fig. 3b; Supplementary Figure 5c). We further explored nanoscale localization of DYNAMO1 by whole-mount negative-staining immunoelectron microscopy (Fig. 3c). The ultrastructure of the DB ring was defined by a Dnm1-labeled curved amorphous string that was partially peeled away from a rigid ring-shaped skeletal filamentous ring upon treatment with a nonionic detergent ${ }^{17}$. DYNAMO1-labeled $10 \mathrm{~nm}$ immunogold particles were detected on the string of the DB ring, but not on the skeletal filamentous ring, indicating that DYNAMO1 localizes on the DB ring formed at the cytosolic side of the division plane. These results strongly suggest that DYNAMO1 is a component of $\mathrm{MD}$ and POD machineries and generates GTP on their respective Dnm1-based rings.

GTP-generating reaction of DYNAMO1 induces membrane fission. To understand molecular function of DYNAMO1 on the MD and POD machinery, genetic disruption of DYNAMO1, particularly abolishment of nucleoside diphosphate kinase activity is needed. Nucleotide diphosphate kinases form an oligomer, including DYNAMO1 (Supplementary Figure $2 \mathrm{j}$ and $\mathrm{k}$ ), and various dominant negative forms have been reported ${ }^{31-33}$. Thus, we next suppressed DYNAMO1 functions by transient expression of DYNAMO1 H116D, a DYNAMO1 mutant defective for GTPgenerating activity (Fig. 1e). DYNAMO1-HA and DYNAMO1 H116D-HA were expressed at about twofold higher than the endogenous protein (Supplementary Figure $6 \mathrm{a}$ and b). Overexpression of DYNAMO1-HA did not affect the division of mitochondria or peroxisomes (Supplementary Figure $6 c$ and d), whereas cells expressing DYNAMO1 H116D-HA showed reductions in division of mitochondria and peroxisomes to an extent similar to that in Dnm1-knockdown cells (Fig. 4a, e; Supplementary Figure 6e-j). Thus, DYNAMO1 H116D apparently functions in a dominant negative fashion. In DYNAMO1 H116D-expressing cells, DYNAMO1 H116D-HA and Dnm1 were properly recruited to the division sites of mitochondria (Fig. 4b; Supplementary Figure 7a and b). However, DYNAMO1
H116D-HA-positive mitochondria were elongated asymmetrically by $1.5-2.0$-fold longer than normal mitochondria and exhibited completely impaired constriction, hence appearing as hyper-constricted mitochondria (Fig. 4b-d; Supplementary Figure 7c and d). Similarly, DYNAMO1 H116D-HA-positive peroxisomes had a hyper-constricted phenotype, which were 2-3fold longer than normal peroxisomes and exhibited stalled constriction (Fig. 4f-h; Supplementary Figure 7e-h). Thus, the H116D mutation impairs complete fission of these two types of organelles. Moreover, in cells expressing antisense-DYNAMO1, mitochondria were not constricted and Dnm1 assembly at the division site was significantly reduced (Supplementary Figure 7i-1). Collectively, these data suggest that the GTPgenerating activity of DYNAMO1 is essential for complete constriction at organelle division sites, and that adequate amounts of DYNAMO1 appear to be required for Dnm1 recruitment. DYNAMO1 localized to the division sites of both organelles and the entire cytoplasm, suggesting two possibilities of the GTPproviding mechanism. One is local GTP generation at the Dnm1positive organelle division site and the other is direct regulation of mitochondrial and peroxisomal divisions using a GTP pool in the cytosol. Although DYNAMO1 was localized at organelle division sites and in the entire cytoplasm (Fig. 2), protein translation is likely normal under the expression of DYNAMO1 H116D (Supplementary Figure 8a and b). Thus, inhibition of organelle divisions is supposed to be caused by local GTP generation at organelle division sites rather than depletion of the cytosolic GTP pool. However, DYNAMO1 H116D expression impaired cell cycle progression (Supplementary Figure 8d). Similarly, inhibition of mitochondrial and peroxisomal divisions by supressing Dnm1 also impaired cell cycle progression (Supplementary Figure 8c-e). Therefore, even at a normal cytosolic GTP level, cell cycle progression might be stalled when there are abnormal divisions of mitochondria and peroxisomes, similar to prophase arrest induced by inhibition of plastid division ${ }^{34}$. Interestingly, plastid division occurred under inhibition of DYNAMO1 (Supplementary Figure 8f), although it is also mediated by GTPase dynamin-like protein Dnm2, a homolog of Dnm1 ${ }^{35}$. Thus, DYNAMO1 is specifically involved in the divisions of both mitochondria and peroxisomes. In C. merolae, at least two genes encode nucleoside diphosphate kinases including DYNAMO1 and its homolog, CMK060c ${ }^{14}$. CMK060c possibly accounts for homeostasis of GTP generation, such as sustaining the global GTP level or compensating for DYNAMO1 functions in the cytosol.

DYNAMO1 facilitates constriction of Dnm1-based ring. To further investigate mechanisms underlying DYNAMO1 functions in the constriction of mitochondria and peroxisomes, we established an in vitro constriction assay using whole-mount negativestaining electron microscopy. Upon incubation of recombinant DYNAMO1 with Dnm1 under various conditions, single strings (DYNAMO1-Dnm1 strings) were formed with a semi-ring structure and diameter of $\sim 200 \mathrm{~nm}$ (Fig. 5a). The strings 
a

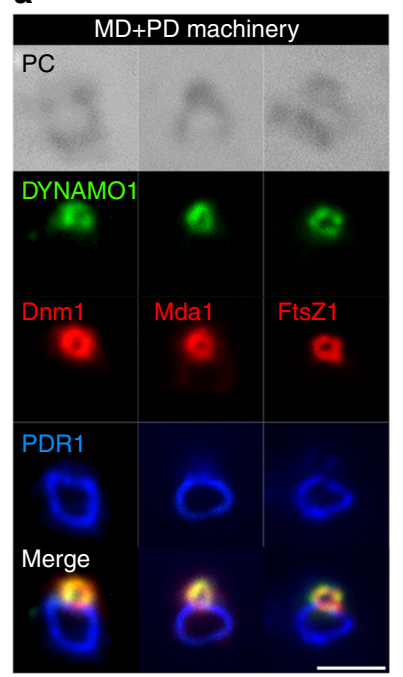

b

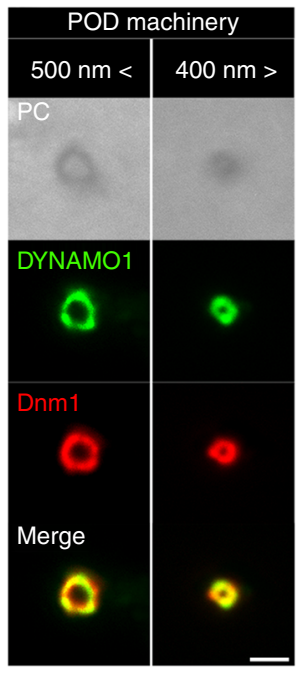

C

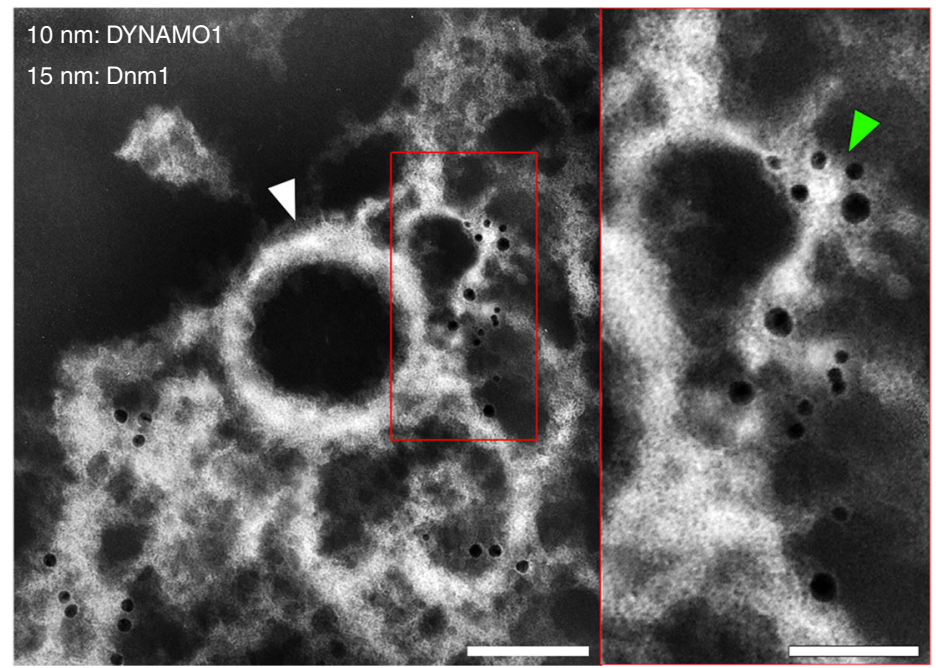

Fig. 3 Localization of DYNAMO1 in MD and POD machineries. a Phase contrast and immunofluorescence microscopy images of DYNAMO1, Dnm1, Mda1 (anti-Mda1 antibody), FtsZ1 (anti-FtsZ1-antibody), and PDR1 (marker protein for PD machinery, anti-PDR1-antibody) on an isolated MD/PD machinery complex. b Phase contrast and immunofluorescence microscopy images of DYNAMO1 and Dnm1 on isolated POD machineries with diameters of $>500$ and $<400 \mathrm{~nm}$. c Whole-mount negative-staining immunoelectron microscopy images of DYNAMO1 (10 nm immunogold particles) and Dnm1 (15 nm immunogold particles) on isolated POD machinery. Right panel shows a magnified image of the red-boxed area in the left panel. The white arrowhead indicates a filamentous ring serving as a skeletal structure of POD machinery. The green arrowhead indicates a DB ring generating motive force for constriction. Scale bars: $1 \mu \mathrm{m}$ (a); $500 \mathrm{~nm}$ (b); $200 \mathrm{~nm}$ (c); $50 \mathrm{~nm}$ (magnified image in c)

contained both DYNAMO1 and Dnm1 as assessed by immunofluorescence microscopy (Fig. 5a, lower panels; Supplementary Figure 9a). Substitution of DYNAMO1-free Dnm1 led to significantly less string formation and short filament-like structures with diameter of $\sim 10-20 \mathrm{~nm}$ were observed (Fig. 5b; Supplementary Figure 9b). String formation was maximized when the amount of DYNAMO1 molecules was half that of Dnm1 (Supplementary Figure 9c). This result supported the observation that downregulation of DYNAMO1 decreased Dnm1 assembly at mitochondrial division sites (Supplementary Figure 7i-l). Dnm1 of $C$. merolae forms a tetramer with a diameter of $\sim 10 \mathrm{~nm}$ in offmembrane state in vitro ${ }^{36}$, and Dnm1 molecules in the division machineries are most likely recruited from membrane-free Dnm1 pool within cytosol ${ }^{19,25}$. Thus, the presence of DYNAMO1 likely induces conformational changes to organize Dnml molecules into a highly ordered ring-like structure or stabilizes a ring-like structure at the division site. Because DYNAMO1 showed a GTPgenerating activity when supplied with both ATP and GDP (Fig. 1e), we verified whether this enzymatic activity was required for the formation of DYNAMO1-Dnm1 strings. Upon addition of ATP and GDP, the morphology of DYNAMO1-Dnm1 strings was dramatically altered to a spiral shape with one or both tips constricted toward the inside of the string (Fig. $5 c-f$ ). The same result was observed with addition of GTP (Fig. $5 \mathrm{~d}, \mathrm{~g}$ ). In contrast, addition of GMP-PCP did not induce the constriction activity (Fig. 5d, h). Thus, constriction of DYNAMO1-Dnm1 strings depended on the GTPase activity of Dnm1. DYNAMO1-free Dnm1 strings showed little evidence of this structural alteration (Fig. 5d). Collectively, these data indicate that DYNAMO1 is involved in facilitating constriction of the string. When the DYNAMO1-Dnm1 string was replaced by a DYNAMO1 H116DDnm1 string (Supplementary Figure 9a), there was no constriction upon addition of ATP or GDP (Fig. 5d, i). However, the DYNAMO1 H116D-Dnm1 string was constricted upon addition of GTP (Fig. 5d, j). Thus, the DYNAMO1 function in constriction of DYNAMO1-Dnm1 strings was independent of the GTPgenerating activity. Because the GTPase activity of Dnm1 was higher in DYNAMO1-Dnm1 and DYNAMO1 H116D-Dnm1 strings, in which DYNAMO1 contained half the amount of Dnm1, compared with DYNAMO1-free Dnm1 strings (Supplementary Figure 10), efficient GTP hydrolysis may have generated strong constrictive forces in the DYNAMO1-Dnm1 strings. GTPase activities of Dnm1 and dynamin are increased in an assembly-dependent manner ${ }^{8,9}$. Therefore, DYNAMO1-mediated stabilization of Dnm1 strings likely facilitates GTPase activity of the rings. Taken together, whole-mount morphological observations demonstrated three different DYNAMO1 functions (Fig. 5k): (1) DYNAMO1 enhances formation of the highly ordered structure of Dnm1; (2) DYNAMO1 generates GTP from ATP and GDP adjacent to Dnm1 molecules; (3) DYNAMO1 constructs DYNAMO1-Dnm1 strings that will then be constricted upon hydrolysis of GTP by Dnm1.

\section{Discussion}

Here, we demonstrated that a novel component, DYNAMO1, of $\mathrm{MD}$ and POD machineries is an essential molecule for the division of both mitochondria and peroxisomes. We propose three functions of DYNAMO1 in driving these organelle divisions in our working model (Fig. 6). First, the DYNAMO1 molecule is a constituent of MD and POD machineries. DYNAMO1 forms a complex structure with Dnm1 and is involved in Dnm1 assembly at the division site (Phase 1). Second, DYNAMO1 generates GTP on the rings of $\mathrm{MD}$ and $\mathrm{POD}$ machineries, serving as the energy source most likely provided by consumption of cytosolic ATP for constriction of organelle division machineries (Phase 2). Finally, DYNAMO1 drives constriction of $\mathrm{MD}$ and POD machineries (Phase 3). The NDPK domain in DYNAMO1 is conserved among eukaryotes. In flies, dysfunction of nucleoside diphosphate kinase (NDPK/NME) identified in the Awd mutant enhances paralysis of the Shibire mutant, a temperature-sensitive mutant of dyna$\min ^{37}$. In mammalian cells, an NDPK ortholog, NME1/NME2, physically interacts with dynamin at the clathrin-coated endocytic pit and facilitates receptor-mediated endocytosis ${ }^{27,28}$. Moreover, NME4 supports dynamin-related GTPase OPA1 that mediates inner mitochondrial membrane fusion ${ }^{29,30}$. Here, we showed that 
a

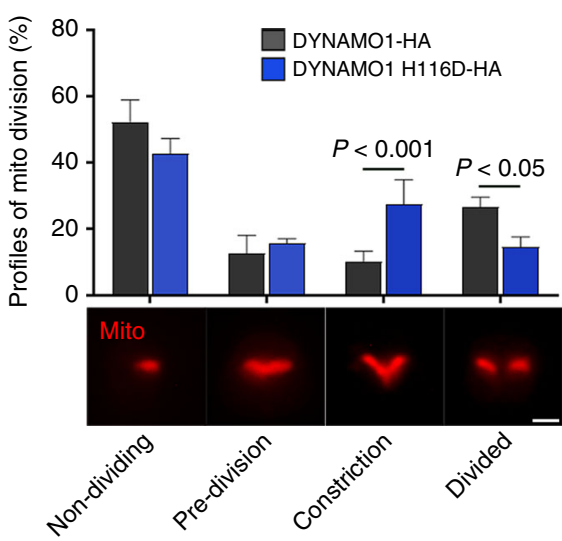

e

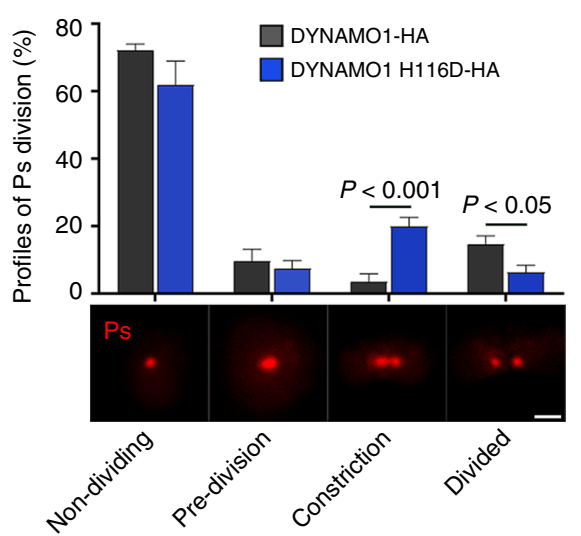

b

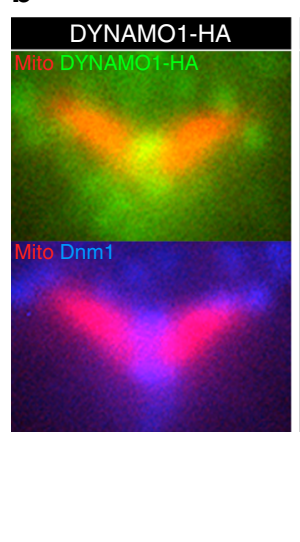

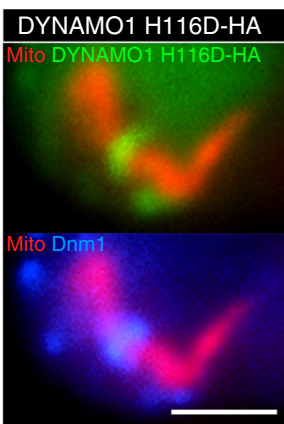

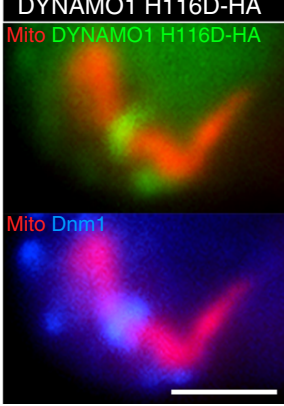

f
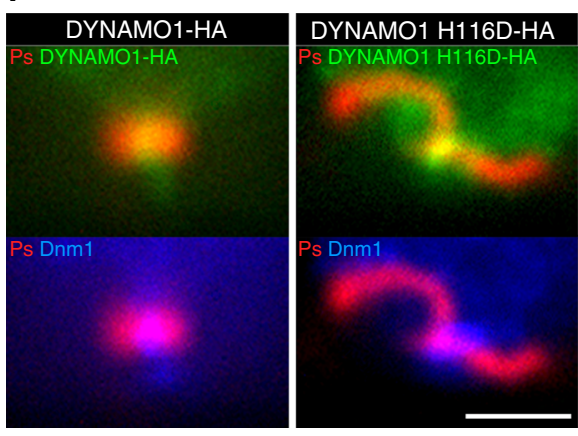

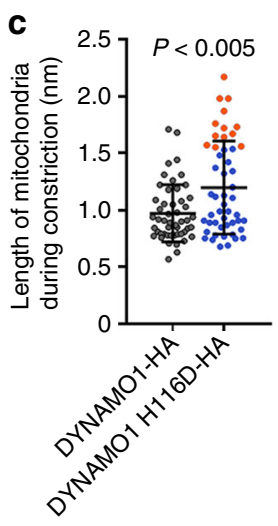

g

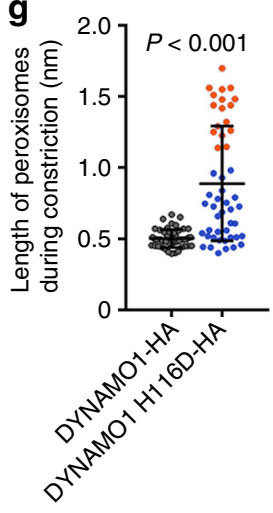

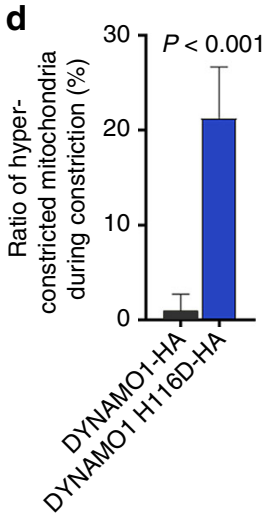

h

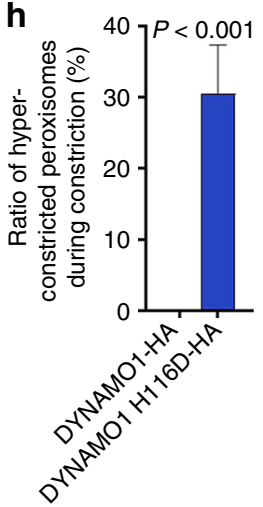

Fig. 4 Disruption of DYNAMO1 functions and whole-mount negative-staining electron microscopy analysis of DYNAMO1-Dnm1 dynamics. a Profiles of mitochondrial division based on morphological changes during division. Mitochondria are labeled as in Fig. 2 a. $n=3$, at least 50 cells were counted in each experiment. b Localization of expressed DYNAMO1-HA and DYNAMO1 H116D-HA (Alexa 488-conjugated anti-HA antibody), Dnm1, and mitochondria in metaphase cells. c Length of mitochondria in cells expressing DYNAMO1-HA or DYNAMO1 H116D-HA. $n=50$ in each experiment. Orange dots indicate hyper-constricted mitochondria as determined by quantification of their length that was elongated by more than $1.5 \mu \mathrm{m}$, which were positive for DYNAMO1 and Dnm1. d Ratio of hyper-constricted mitochondria during the constriction phase. e Profiles of peroxisomal division based on morphological changes during division. $n=3$, at least 50 cells were counted in each experiment. $\mathbf{f}$ Localization of DYNAMO1-HA, DYNAMO1 H116D-HA, Dnm1, and peroxisomes in anaphase cells. $\mathbf{g}$ Lengths of peroxisomes in cells expressing DYNAMO1-HA or DYNAMO1 H116D-HA. $n=50$ in each experiment. Orange dots indicate hyper-constricted peroxisomes as determined in $\mathbf{c}$ by quantification of their length that was elongated by more than $1 \mu \mathrm{m}$, which were positive for DYNAMO1 and Dnm1. h Ratio of hyper-constricted peroxisomes during the constriction phase. Scale bars: $1 \mu$ m. Data are means \pm s.d. $P ; p$-value (Mann-Whitney test)

the NDPK ortholog DYNAMO1 is a component of the Dnm1based organelle division machinery of mitochondria and peroxisomes, which most likely supports local GTP generation to induce the constrictive force. Thus, local GTP generation by the dynamin family member during membrane remodeling is a conserved phenomenon among eukaryotes. Although dynamin family members require considerable amounts of GTP because of the high rate of GTP hydrolysis and lower affinity for $\mathrm{GTP}^{7-9}$, cellular free-GTP levels are low $(150-300 \mu \mathrm{M})^{38,39}$. The local enrichment of GTP is potentially critical to provide a sufficient energy source to drive the supramolecular nanomachineries that influence the dynamic behavior of organelles and cellular structures.

\section{Methods}

Isolation of MD/PD and POD machineries. For synchronization, C. merolae 10D cells were grown in $2 \times$ Allen's medium. First, cells were subcultured at $1 \times 10^{7}$ cells $/ \mathrm{mL}$ in $500 \mathrm{~mL}$ flat bottles and subjected to a 12-h light/12-h dark cycle (100 $\mu \mathrm{Em}^{2} / \mathrm{s}$ ) at $42^{\circ} \mathrm{C}$ under aeration with ordinary air ${ }^{40}$. To isolate MD machinery, synchronized cells were harvested at $13 \mathrm{~h}$ after the one set of synchronization. To isolate POD machinery, synchronized cells were cultured under additional treatment with a $1 / 2500$ volume of $100 \mathrm{mM}$ oryzalin stock solution dissolved in dimethyl sulfoxide at $8 \mathrm{~h}$ after the one set of synchronization to increase the yield of dividing peroxisomes, then cells were collected at $20 \mathrm{~h}$ the one set of synchronization ${ }^{17}$. Harvested cells were centrifuged at $750 \times g$ for $5 \mathrm{~min}$ and resuspended in isolation medium A (20 mM Tris. $\mathrm{HCl}, \mathrm{pH} 7.6,5 \mathrm{mM} \mathrm{MgCl}_{2}, 30 \mathrm{mM} \mathrm{KCl}, 5 \mathrm{mM}$ EGTA, $1 \mathrm{mM}$ dithiothreitol (DTT), $3 \mathrm{mg} / \mathrm{mL}$ cOmplete EDTA-free protease inhibitor mixture (hereafter cOmplete) (Roche), and $0.18 \mathrm{M}$ sucrose). Resuspended cells were incubated for $30 \mathrm{~min}$ at $42^{\circ} \mathrm{C}$ in the dark condition. For subcellular fractionation, cells were homogenized using a French Pressure Cell (SLM Aminco) at $600 \mathrm{psi}$. The homogenate was added with $100 \mu \mathrm{g} / \mathrm{mL}$ DNase I and incubated for $1 \mathrm{~h}$ on ice. For the isolation of POD machinery, $0.5 \% \mathrm{wt} / \mathrm{vol}$ Triton X-100 was added. The homogenate $(20 \mathrm{~mL})$ were loaded on a Percoll gradient $(4 \mathrm{~mL}$ of $80 \%$ Percoll, $8 \mathrm{~mL}$ of $60 \%$ Percoll, $6 \mathrm{~mL}$ of $40 \%$ Percoll, and $2 \mathrm{~mL}$ of $0 \%$ Percoll). Percoll was dissolved in isolation medium B (20 mM Tris. $\mathrm{HCl}, \mathrm{pH} 7.6,5 \mathrm{mM} \mathrm{MgCl}, 30$ $\mathrm{mM} \mathrm{KCl}, 5 \mathrm{mM}$ EGTA, $1 \mathrm{mM}$ DTT, $3 \mathrm{mg} / \mathrm{mL}$ cOmplete, and $0.3 \mathrm{M}$ sucrose). After centrifugation at $23,000 \times g$ for $60 \mathrm{~min}, 2 \mathrm{~mL}$ of the organelle fraction at the top of the $40-60 \%$ Percoll surface was collected as organelle fraction and then the upper $10 \mathrm{~mL}$ was collected as the cytosolic fraction, hence concentration ratio between organelle fraction and cytosolic fraction is about 5 to 1 . Organelle fraction was washed twice with isolation medium $\mathrm{B}$ without sucrose and incubated on ice for 30 min in $15 \mathrm{~mL}$ of isolation medium C (20 mM Tris. $\mathrm{HCl}, \mathrm{pH} 7.6,5 \mathrm{mM} \mathrm{MgCl}_{2}, 30$ $\mathrm{mM} \mathrm{KCl}, 5 \mathrm{mM}$ EGTA, $1 \mathrm{mM}$ DTT, $3 \mathrm{mg} / \mathrm{mL}$ cOmplete, $1 \%$ Nonidet-P40) on ice for $30 \mathrm{~min}$. The fraction was layered on a $15 \mathrm{~mL}$ of $40 \%$ (vol/vol) Percoll and 
a

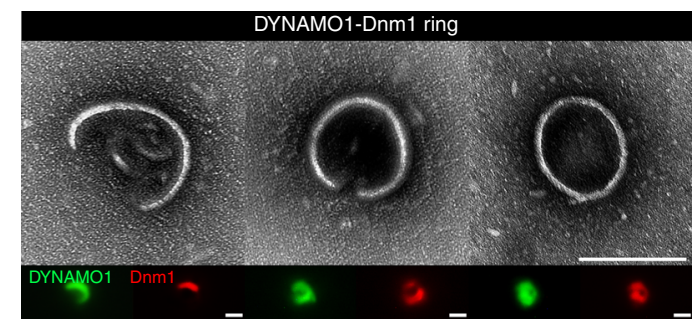

C

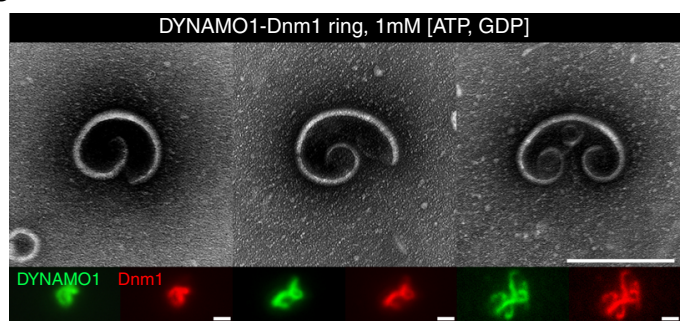

d

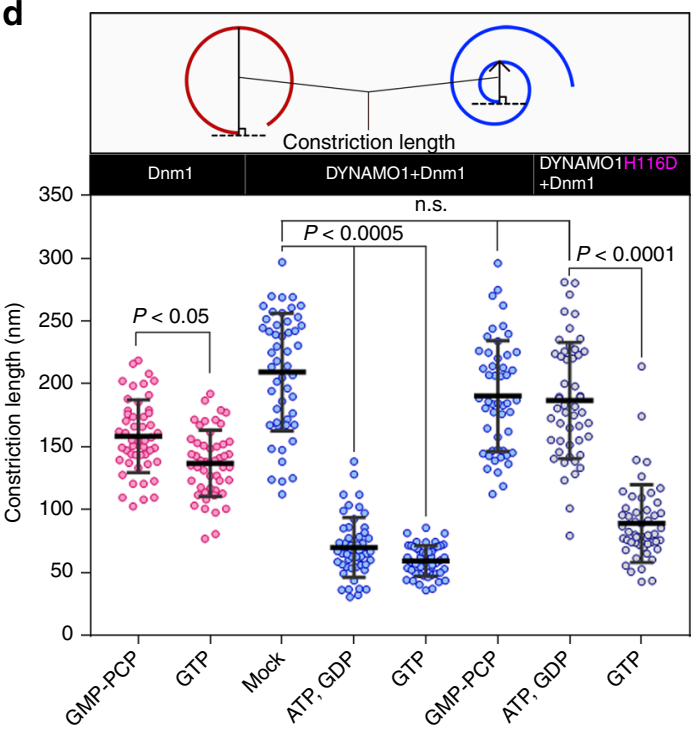

b

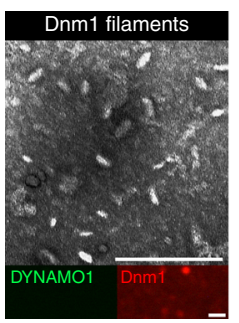

e

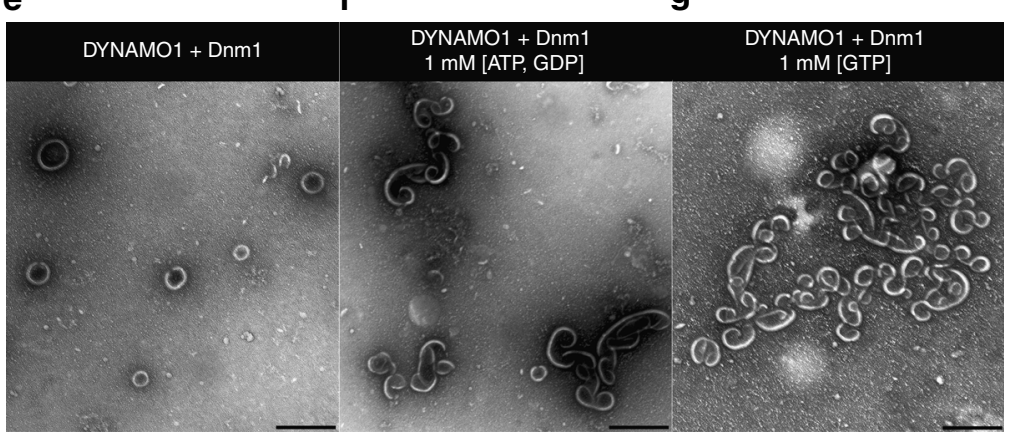

h

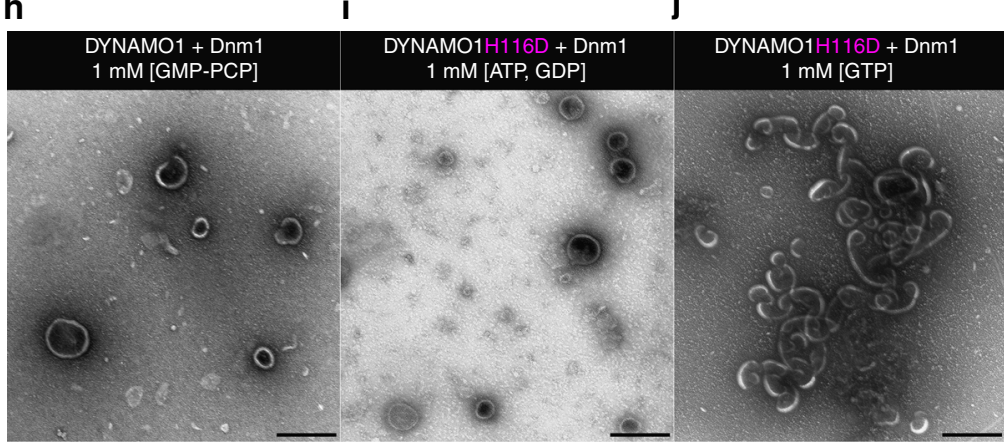

$\mathbf{k}$

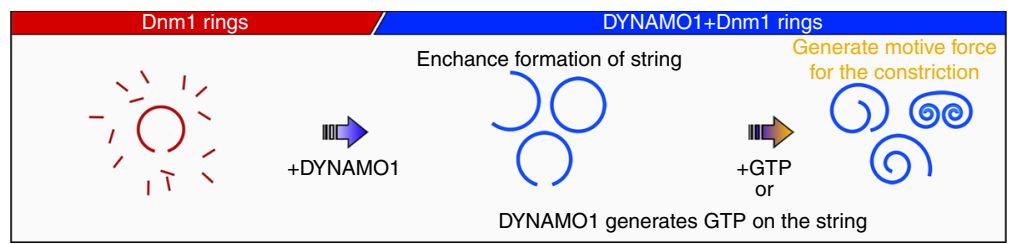

Fig. 5 Structure of DYNAMO1-Dnm1 string. a Typical structure of DYNAMO1-Dnm1 strings under GTP-free conditions. Immunofluorescence images of DYNAMO1 (green) and Dnm1 (red) are shown in the respective lower panels. b Typical structure of DYNAMO1-free Dnm1 filaments and strings under GTP-free condition (left, three panels) or in the presence of GTP (right panel). Immunofluorescence images are shown in the lower panels. c Typical structure of DYNAMO1-Dnm1 strings with addition of ATP and GDP. Immunofluorescence images are shown in the lower panels. d Schematic image representing measurement of constriction lengths of the strings. Constriction lengths of the DYNAMO1-free Dnm1, DYNAMO1-Dnm1, and DYNAMO1 H116D-Dnm1 strings under the various conditions $(n=50)$. e-j Structural dynamics of DYNAMO1-Dnm1 or DYNAMO1 H116D-Dnm1 strings upon addition of ATP and GDP, GTP, or GMP-PCP. $\mathbf{k}$ Schematic image representing the dynamics of DYNAMO1-Dnm1 strings. Scale bars: $200 \mathrm{~nm}$ (a-c); $500 \mathrm{~nm}$ (e-j). Data are means \pm s.d. n.s. not significant. $P$; $p$-value (unpaired $t$-test for Dnm1 and DYNAMO1 H116D + Dnm1, Kruskal-Wallis nonparametric ANOVA for DYNAMO1 + Dnm1)

centrifuged at $18,000 \times g$ for $30 \mathrm{~min}$. To isolate $\mathrm{MD} / \mathrm{PD}$ machineries, $1.5 \mathrm{~mL}$ of the Percoll surface was collected and concentrated to $200 \mu \mathrm{L}$ in isolation medium C by spinning down. To isolate POD machineries, $1.5 \mathrm{~mL}$ of the Percoll surface was mixed vigorously using by vortex and mixed with Opti-prep at final concentration of $42.3 \%$. Then, fraction was centrifuged at $200,000 \mathrm{rpm}$ for $3 \mathrm{~h}$ in a NVT65.2 rotor (Beckman Instruments). Fraction $1.0 \mathrm{~mL}-3.0 \mathrm{~mL}$ from the bottom was collected after the centrifugation and scaled down into $200 \mu \mathrm{L}$ in isolation medium $\mathrm{C}$ by spinning down. Collected MD/PD or POD fractions were then mixed with detergents, LDAO (Sigma-Aldrich) and SDS, at final concentration $0.2 \%$ and $0.05 \%$, respectively. For observation of machineries, this step was performed after the immunostaining on slide glass at room temperature for $1 \mathrm{~min}$. Protein expression levels of DYNAMO1 in membrane and cytosol fractions were quantified by SDS-PAGE and western blotting using ImageJ. Intensities were calculated by subtracting the background intensity of each lane from the DYNAMO1 intensity. The intensities of DYNAMO1 were normalized to whole cell lysate based on how much concentrated during the fractionation described above.

Sequences for multiple alignment. The sequences used for multiple alignment were collected by BLAST searches in the databases of the respective species, National Centre for Biotechnology Information, the US Department of Energy Joint Genome Institute (http://genome.jgi-psf.org/), using DYNAMO1 (CML110) of the red alga C. merolae as the query. DYNAMO1 (C. merolae, CMC110C); DYNAMO1 isoform (C. merolae, CMK060C); Thalassiosira pseudonana, XP_002295246.1; Dictyostelium discoideum, XP_644519.1; Schisosaccharomyces pombe, NP_592857.1; Saccharomyces cerevisiae, NP_012856.1; Arabidopsis 


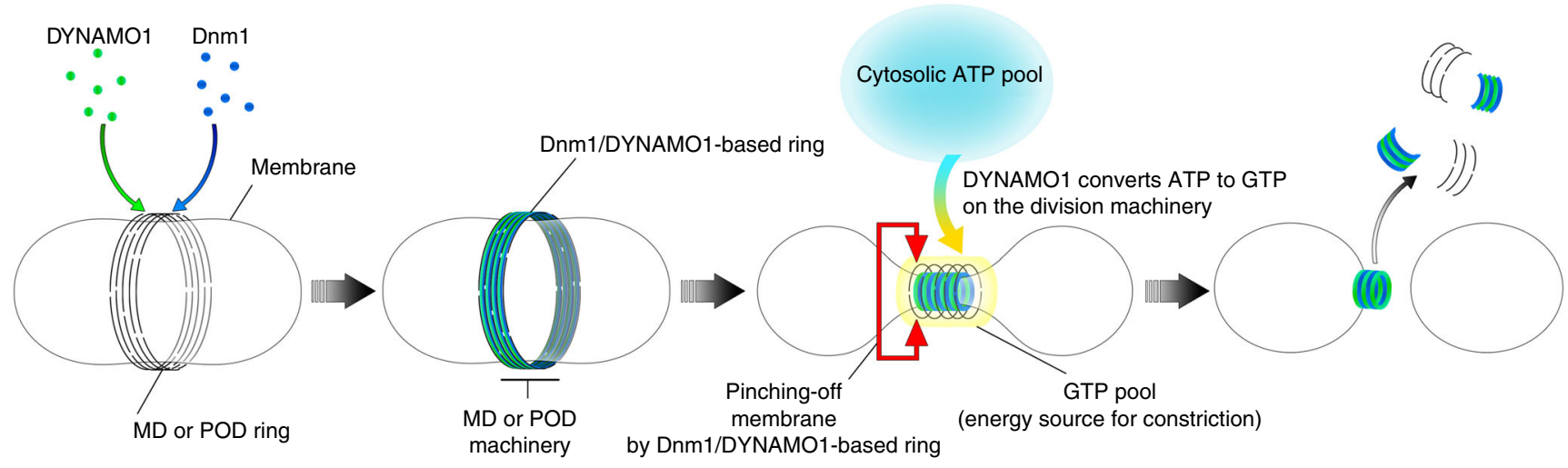

Phase 1

Assembly of Dnm1/DYNAMO1-based ring

DYNAMO1 enhances Dnm1

assembly around the division site
Phase 2

Constriction of membrane by DYNAMO1 and Dnm1 activities

DYNAMO1 generates GTP via energy conversion of ATP

DYNAMO1 facilitates Dnm1/DYNAMO1-based ring to generate strong constriction force

Fig. 6 A working model of DYNAMO1 functions during the division of mitochondrion and peroxisomes. Initially, DYNAMO1 is recruited to the division site of the mitochondrion, together with Dnm1 (Phase 1, prophase). DYNAMO1 is involved in Dnm1 assembly around the division site. Next, DYNAMO1 converts ATP to GTP on the ring of the MD machinery. A cytosolic ATP source is used for this reaction. Upon GTP generation, the MD machinery is constricted and GTPase Dnm1 pinches off mitochondrion. Moreover, the DYNAMO1-Dnm1 structure effectively generates a strong motive force for the constriction (Phase 2). MD machinery containing DYNAMO1 and Dnm1 is immediately disassembled (Phase 3). After mitochondrial division, DYNAMO1 functions in the POD machinery during meta-anaphase, in an analogous manner to that in mitochondrial division

thaliana, NP_567346.2; Caenorhabditis elegans, NP_492761.1; Drosophila melanogaster, NP_476761.3; Mus musculus, NP_032730.1, and Homo sapiens, NP_937818.1 were aligned.

Antibodies used for this study. To generate anti-DYNAMO1 antisera in rabbits, mice and rat, the open reading frame of CML110C protein from C. merolae was amplified by PCR using the following primers: $5^{\prime}$-cgcGGAyTCCatggaggagacgactttcatcatg- $3^{\prime}$ and $5^{\prime}$-cgcCTCGAGagaaacgttctcgtagacccagc- $3^{\prime}$ (Bam $\mathrm{HI}$ and XhoI sites are capitalized, respectively). The amplified DNA fragment was cloned into the BamHI and XhoI sites of pGEX6p-1 after restriction digestion. The resulting recombinant protein was purified on a GSTrap HP column (GE Healthcare) and subcutaneously injected into rabbits, mice, or rats for immunization (T.K. Craft Corp.). DYNAMO1 antibodies are used for immunofluorescence microscopy (1/3000 dilution) and for immunoblotting (1/2000 dilution). The other antibodies used in this study were a rabbit anti-Dnml antibody ${ }^{19}(1 / 2000$ dilution for immunofluorescence microscopy and immunoblotting), mouse Mdal antibody $^{41}$ (1/400 for immunofluorescence microscopy and 1/1000 for immunoblotting), rat EF-Tu antibody ${ }^{42}$ (1/1000 for immunofluorescence microscopy and immunoblotting), mouse FtsZ1 antibody ${ }^{20}$ (1/100 for immunofluorescence microscopy), guinea pig anti-EF1 $\alpha$ antibody ${ }^{43}$ (1/2000 for immunoblotting), guinea pig anti-porin antibody ${ }^{44}$ (1/400 for immunoblotting), rat anti-PDR1 antibody ${ }^{21}$ (1/200 for immunofluorescence microscopy), and rabbit Pex14 antibody ${ }^{25}$ (1/1000 for immunoblotting). The Alexa 488-conjugated mouse anti-HA antibody was purchased from Thermo Fisher Scientific (Cat. \#A-21287). A mouse anti-GFP antibody (B2) was purchased from Santa Cruz Biotechnology (Cat. \#sc-9996). For immunoblotting, the secondary antibody was alkaline phosphatase-conjugated rabbit and mouse IgG (BioRad). Original data of immunoblotting with these antibodies are shown in Supplementary Figure 11. For immunofluorescence microscopy, the secondary antibodies were Alexa Fluor 350- (mouse; Cat. \#A11045, rabbit; Cat. \#A-21068, rat; Cat. \#A-21093) (1/200 dilution), Alexa Fluor 488-(mouse; Cat. \#A-11001, rabbit; Cat. \#A-11008, rat; Cat. \#A-11006) (1/1,000 dilution) and Alexa Fluor 555-(mouse; Cat. \#A-21322, rabbit; Cat. \#A-21428, rat; Cat. \#A-21434) (1/1,000 dilution) conjugated IgGs (Thermo Fisher Scientific). For immunoelectron microscopy, mouse and rabbit 10-nm (Cat. \#810.177) (1/40 dilution) or 15-nm (Cat. \#815.166) (1/20 dilution) immunogold particleconjugated IgGs were purchased from Aurion.

Plasmid construction. To construct a plasmid for expression of recombinant Dnml protein, the Dnm1 (CME019) sequence was amplified from C. merolae genomic DNA with the following primers: $5^{\prime}$-cgcGGATCCatggagcgcctaatacctatcg$3^{\prime}$ (BamHI site is capitalized) and 5'-cgcGAATTC aatctcctctttgacgtgaacg-3' (EcoRI site is capitalized). The amplified fragment was digested with BamHI and EcoRI (Nippon Gene) and inserted into pGEX6p-1. To prepare a plasmid for transient gene expression, the DYNAMO1 (CML110) sequence, including the upstream $\sim 500$ bp sequence, was amplified with the following primers: $5^{\prime}$-ctctcgaggcagaggaatgtggacagtcag- $3^{\prime}$ and $5^{\prime}$-ttgctcacagaaacgttctcgtagacccagc- $3^{\prime}$. The amplified fragments were combined with pTH-2PL ${ }^{45}$ linearized with primers $5^{\prime}$-acgtttctgtgagcaagggcgaggag- $3^{\prime}$ and $5^{\prime}$-cctctgcctcgagagcttggcactgg- $3^{\prime}$ using an In-Fusion HD cloning kit (Clontech). A triple tandem HA-tagged sequence $(3 \times \mathrm{HA}$-tag) was inserted into the C-terminus of DYNAMO1 by PCR with the following primers: $5^{\prime}$ TATGACGTCCCGGACTATGCAGGATACCCTTATGACGTTCCA-

GATTACGCTtaaatcgttcaaacatttggcaataaag-3' and 5'-AGTCCGGGACGTCATAGGGATAGCCCGCATAGTCAGGAACATCGTATGGGTAagaaacgttctcgtagacccagc- $3^{\prime}(3 \times$ HA-tag is capitalized). The product was circularized using the InFusion HD cloning kit. The antisense-DYNAMO1 sequence was amplified from $C$. merolae genomic DNA with the following primers: $5^{\prime}$-cgcGGATCCgtcattgacgcctcagatagcgtggagag- $3^{\prime}$ (BamHI site is capitalized) and $5^{\prime}$-cgcAAGCTTatggaggagacgactttcatcatg- $3^{\prime}$ (HindIII site is capitalized). The amplified fragment was treated with BamHI and HindIII and inserted into pMito-GFP ${ }^{17}$. The antisense Dnm1 plasmid co-expressing mCherry is described elsewhere ${ }^{25}$. To generate DYNAMO1 with the H116D mutation, a plasmid encoding DYNAMO1 was amplified with the following primers: $5^{\prime}$-gtcattgacgcctcagatagcgtggagag- $3^{\prime}$ and $5^{\prime}$-tgaggcgtcaatgacgtttcgccctacg- $3^{\prime}$. The amplified linear construct harboring His-116 replaced by Asp was recovered using the In-Fusion HD cloning kit, as described for the DYNAMO1 expressing plasmid.

Purification of DYNAMO1. For DYNAMO1 expression, the plasmid encoding GST-tagged DYNAMO1 used for antibody generation was employed as described above. For DYNAMO1 H116D expression, the GST-tagged DYNAMO1 plasmid was amplified with the same primers to generate the plasmid for transient expression of the H116D mutant described above, and the amplified fragment was cloned by infusion cloning. XL-1 Blue strain cells were transformed with these plasmids, cultured at $37^{\circ} \mathrm{C}$ for $12 \mathrm{~h}$ in $100 \mathrm{~mL}$ Luria Bertani (LB) medium, scaled up to $1 \mathrm{~L} \mathrm{LB}$ medium, and incubated further at $37^{\circ} \mathrm{C}$ for $2 \mathrm{~h}$ and then at $18^{\circ} \mathrm{C}$ for 1 h. Isopropyl $\beta$-D-1 thiogalactopyranoside (IPTG) was added at a final concentration of $0.1 \mathrm{mM}$, and cells were harvested after a further $12 \mathrm{~h}$ of incubation at $18^{\circ} \mathrm{C}$ by centrifugation at $1000 \times \mathrm{g}$ for $10 \mathrm{~min}$. Cell pellets were resuspended in $200 \mathrm{~mL}$ HEPES buffer (HB250) containing $250 \mathrm{mM} \mathrm{NaCl}, 20 \mathrm{mM}$ HEPES-KOH, pH 7.5, 2 $\mathrm{mM}$ EGTA, $1 \mathrm{mM} \mathrm{MgCl} 2,1 \mathrm{mM}$ DTT, and a cOmplete. After homogenization by sonication for $10 \mathrm{~min}$, the supernatant was filtered and rotated at $4^{\circ} \mathrm{C}$ for $1 \mathrm{~h}$ with $1 \mathrm{~mL}$ glutathione-Sepharose $4 \mathrm{~B}$ beads (GE Healthcare). The beads were collected, washed, and resuspended in $10 \mathrm{~mL} \mathrm{HB250}$. Then, the beads were loaded onto a 10 $\mathrm{mL}$ Poly-Prep Chromatography column (BioRad) and washed with HBs: $5 \mathrm{~mL}$ HB250, $10 \mathrm{~mL} \mathrm{HB100} \mathrm{containing} 100 \mathrm{mM} \mathrm{NaCl}$, and $20 \mathrm{~mL}$ cOmplete-free HB50 containing $50 \mathrm{mM} \mathrm{NaCl}$. To remove GST-tag, Sepharose beads were treated with PreScission Protease (GE Healthcare) at $4{ }^{\circ} \mathrm{C}$ for 2 days, and DYNAMO1 was eluted. 
Purification of Dnm1. For Dnm1 expression, pGex6p-1Dnm1 ${ }^{25}$ was introduced into the Escherichia coli XL-1 Blue strain. Transformed XL-1 Blue strain cells were cultured at $37^{\circ} \mathrm{C}$ for $12 \mathrm{~h}$ in $100 \mathrm{~mL}$ of LB medium, scaled up to $1 \mathrm{~L}$ of LB medium, and further incubated at $37^{\circ} \mathrm{C}$ for $2 \mathrm{~h}$ and at $18{ }^{\circ} \mathrm{C}$ for $1 \mathrm{~h}$. IPTG was added to a final concentration of $0.1 \mathrm{mM}$, and cells were harvested after a further 8 -h incubation at 18 ${ }^{\circ} \mathrm{C}$ by centrifugation at $1000 \times \mathrm{g}$ for $10 \mathrm{~min}$. Cell pellets were resuspended in $100 \mathrm{~mL}$ of HB150. After homogenization by sonication, the supernatant was filtered and rotated at $4^{\circ} \mathrm{C}$ with $1 \mathrm{~mL}$ of glutathione Sepharose $4 \mathrm{~B}$ beads (GE Healthcare) for $1 \mathrm{~h}$. The beads were collected and resuspended in $10 \mathrm{~mL}$ of HB100. Then, beads were loaded onto a $10 \mathrm{~mL}$ Poly-Prep Chromatography column (BioRad) and washed with cOmplete-free HB75 and $20 \mathrm{~mL}$ of complete-free HDB50. After washing with HBs, Sepharose beads were treated with PreScission Protease (GE Healthcare) at $4{ }^{\circ} \mathrm{C}$ for 2 days and the cleaved-off proteins were eluted.

Gel filtration assay of recombinant DYNAMO1. A total of $1 \mu \mathrm{g}$ purified recombinant DYNAMO1 was analyzed by gel filtration on a Sepharose 6 10/300 GL column using an AKTA prime system (GE Healthcare). Gel filtration standards (BioRad Laboratories) including thyroglobulin $(669 \mathrm{kDa}), \gamma$-globulin $(158 \mathrm{kDa})$, and ovalbumin $(45 \mathrm{kDa})$ were used to calculate the molecular mass of DYNAMO1. Partition coefficient $K_{\mathrm{av}}$ was calculated by

$$
\frac{\left(V_{\mathrm{e}(\mathrm{B})}-V_{0}\right)}{V_{\mathrm{c}}-V_{0}}=K_{\mathrm{av}(\mathrm{B})}
$$

where $V_{\mathrm{e}(\mathrm{B})}$ is the elution volume, $V_{0}$ is the void volume, and $V_{\mathrm{c}}$ is the total geometric volume of the column, $24 \mathrm{~mL}$.

Pull-down assay and immunoprecipitation. Synchronized cells at $\mathrm{M}$ phase $(16 \mathrm{~h}$ after the one set of light/dark cycle) were lysed in binding buffer (50 mM HEPES$\mathrm{KOH}, \mathrm{pH} 7.5,0.15 \mathrm{M} \mathrm{NaCl}, 0.5 \%$ Triton X-100, 1 mM EDTA, 1 mM DTT, a cOmplete). Cell lysates were centrifuged at $20,000 \times g$ for $5 \mathrm{~min}$ at $4{ }^{\circ} \mathrm{C}$ to remove insoluble cell debris and resulting supernatants were pre-incubated with glutathione-Sepharose beads (GE Healthcare) or protein A Sepharose CL-4B (GE Healthcare) for $1 \mathrm{~h}$ at $4{ }^{\circ} \mathrm{C}$ to reduce nonspecific binding to the Sepharose beads. For pull-down assay, the supernatants or recombinant Dnml were incubated with purified GST-DYNAMO1 for $3 \mathrm{~h}$ at $4{ }^{\circ} \mathrm{C}$. The protein complexes were recovered by incubating for $1 \mathrm{~h}$ at $4^{\circ} \mathrm{C}$ with glutathione-Sepharose beads and eluted with Laemmli sample buffer. For immunoprecipitation, the supernatants were incubated with anti-Dnm1 antibody at $4{ }^{\circ} \mathrm{C}$ for overnight. Antibody-antigen complexes were recovered by incubating for $1 \mathrm{~h}$ at $4{ }^{\circ} \mathrm{C}$ with Protein A-Sepharose CL-4B and eluted with Laemmli sample buffer.

Phase contrast and immunofluorescence microscopies. C. merolae cells were fixed at $-30^{\circ} \mathrm{C}$ for $5 \mathrm{~min}$ with $1 \% \mathrm{wt} / \mathrm{vol}$ paraformaldehyde and $10 \%$ DMSO dissolved in $100 \%$ methanol $^{42}$. Phase contrast and immunofluorescence images were captured with a fluorescence microscope (BX51; Olympus). Immunofluorescence profiles were acquired with ImageJ software (National Institutes of Health, Bethesda, MD, USA).

LC-MS/MS analysis of isolated POD machinery. Proteins in isolated POD machinery and interphase fractions were separated by SDS-PAGE and stained with silver stain $\left(0.02 \% \mathrm{Na}_{2} \mathrm{~S}_{2} \mathrm{O}_{3}, 0.1 \% \mathrm{AgNO}_{3}\right.$, and $0.04 \%$ formalin $/ 2 \% \mathrm{Na}_{2} \mathrm{CO}_{3}$; SigmaAldrich). Signal intensities of silver-stained bands were compared using the Image] plot profile between POD machinery and interphase fractions. Distinct bands with signal intensity ratios higher than 1 of POD machinery to interphase fractions were cut out, reduced with DTT, alkylated with iodoacetamide, digested with trypsin, and analyzed by an ion-trap mass spectrometer equipped with a nano-LC electrospray ionization source at the Human Proteome Research Centre, Kyushu University, Japan ${ }^{46}$. The obtained peptides were dried and then dissolved in a solution containing $0.1 \%$ trifluoroacetic acid and $2 \%$ acetonitrile before nanoscale liquid chromatography (nanoLC)-MS/MS analysis with a system consisting of an LTQ Orbitrap Velos Pro mass spectrometer (Thermo Fisher Scientific) coupled with a nanoLC instrument (Advance LC, Michrom BioResources) and HTC-PAL autosampler (CTC Analytics). Peptide separation was performed with an in-house pulled and fused silica capillary (internal diameter, $0.1 \mathrm{~mm}$; length, $10 \mathrm{~cm}$; tip internal diameter, $0.05 \mathrm{~mm}$ ) packed with a $\mathrm{C} 18 \mathrm{~L}$-column material (average particle size, $3 \mu \mathrm{m}$ ) (Chemicals Evaluation and Research Institute). The mobile phases were $0.1 \%$ formic acid in water (A) and $100 \%$ acetonitrile (B). Peptides were eluted with linear gradients of $5-40 \%$ B for $20 \mathrm{~min}$ and $40-95 \%$ B for $1 \mathrm{~min}$, and then kept at $95 \% \mathrm{~B}$ for $4 \mathrm{~min}$ at a flow rate of $300 \mathrm{~nL} / \mathrm{min}$. Collision-induced dissociation (CID) spectra were acquired automatically in a data-dependent scan mode with the dynamic exclusion option. Full MS spectra were obtained with Orbitrap in the mass/charge $(\mathrm{m} / \mathrm{z})$ range of 300-2000 with a resolution of 60,000 at $\mathrm{m} / \mathrm{z} 400$. The nine most intense precursor ions (minimum ion count threshold of 1000) in the full MS spectra were selected for subsequent ion-trap MS/MS analysis with the automated gain control (AGC) mode. The AGC was set to $1 \times 10^{6}$ for full MS and 1 $\times 10^{4}$ for CID MS/MS. The normalized collision energy value was set to $35 \%$. The lock mass function was activated to minimize mass error during analysis. Peak lists were generated by MSn.exe (Thermo Fisher Scientific) with a minimum scan/group value of 1 and compared with the in-house-curated target/decoy C. merolae genome database (http://merolae.biol.s.u-tokyo.ac.jp/) using the MASCOT algorithm (ver. 2.4.1). Trypsin was selected as the enzyme, the allowed number of missed cleavages was set at 1 , and carbamidomethylation on cysteine was selected as the fixed modification. Oxidized methionine was searched as the variable modification. Precursor mass tolerance was $10 \mathrm{ppm}$ and the tolerance of MS/MS ions was $0.8 \mathrm{Da}$. The threshold used for peptide identification was a MASCOT score of $\geq 30$ and a delta score (the difference between first- and second-assigned peptides) of $\geq 18$. Using these criteria, the false positive rate was $<1 \%$. Contaminants of plastid genome-encoded abundant photosynthetic proteins with a MASCOT score of $\geq 100$ were excluded from the data.

LC-ESI-MS/MS analysis of nucleotides. To measure DYNAMO1 activity for nucleotide exchange, $100 \mathrm{nM}$ recombinant DYNAMO1 described above was mixed with nucleotides and incubated at $37^{\circ} \mathrm{C}$ for $3 \mathrm{~min}$. The reaction mixture was immediately mixed with $1.25 \times$ and $2.5 \times$ volumes of chloroform and methanol, respectively. Subsequently, $2 \mathrm{mM}$ raffinose, as an internal standard used for normalization between samples, was added, and all samples were lyophilized and dissolved in methanol:water $(1: 1 \mathrm{v} / \mathrm{v})$. After centrifugation at $10,000 \times g$ for $5 \mathrm{~min}$, the supernatant was evaporated at room temperature under nitrogen for $6 \mathrm{~h}$ and then subjected to LC-ESI-MS/MS analysis. LC-ESI-MS/MS was performed using a 4000 QTRAP quadrupole linear ion-trap hybrid mass spectrometer (AB Sciex) with an ACQUITY UPLC System (Waters). Samples were injected into an ACQUITY UPLC BEH C18 column $(1 \times 150 \mathrm{~mm}$; Waters $)$ and then directly subjected to ESI-MS/MS analysis. A $10 \mu \mathrm{L}$ aliquot was separated by step gradient elution with mobile phases A (water, $0.1 \%$ formic acid and $0.028 \%$ ammonia) and B ( $20 \%$ acetonitrile, $0.1 \%$ formic acid, and $0.028 \%$ ammonia) at the following ratios: 95:5 (for 0-5 min), 0:100 (5-25 $\mathrm{min}), 0: 100$ (25-30 $\mathrm{min})$, and 95:5 (30-40 min). The flow rate was $50 \mu \mathrm{L} / \mathrm{min}$ at $30^{\circ} \mathrm{C}$. The source temperature was $400^{\circ} \mathrm{C}$, the declustering potential was -85 , and the collision cell exit potential was -11 . The ion transitions at $m / z 506>79,426>79,522>79,442>79$, and $503>179$ for ATP, ADP, GTP, GDP, and raffinose, respectively, were used in the multiple reaction monitoring mode. Data were analyzed and quantified using Analyst software (AB Sciex). Nucleotides were purchased from Sigma-Aldrich.

Electron microscopy. Whole-mount immunoelectron microscopy observations of POD machinery, DYNAMO1 and Dnml structures was performed by transmission election microscopy (Hitachi). The purified proteins were mixed and incubated at $4{ }^{\circ} \mathrm{C}$ overnight and then incubated with various nucleotides at $37^{\circ} \mathrm{C}$ for 5 min. The mixtures were mounted on formvar coated 200-mesh copper grids (Nisshin EM Co., Ltd.) that had been carbonized and glow discharged immediately prior to use. The grids were negatively stained with $2 \%$ uranyl acetate and observed at $80 \mathrm{kV}$. Nucleotides and non-hydrolysable analogs were purchased from SigmaAldrich.

Kinetic analysis of DYNAMO1-Dnm1 complexes. Assays of GTPase activities of DYNAMO1 $(250 \mathrm{nM})$ and Dnm1 $(500 \mathrm{nM})$ were performed at $37^{\circ} \mathrm{C}$ in reaction mixtures (20 mM HEPES, pH 7.5, $30 \mathrm{mM} \mathrm{KCl,} 1 \mathrm{mM} \mathrm{MgCl} 2$ and $1 \mathrm{mM} \mathrm{DTT}$ ). Released phosphate was detected by Malachite Reagent (BioAssay Systems). The absorbance at $600 \mathrm{~nm}$ was measured with a microplate reader (Benchmark Plus).

Quantification and statistical analysis. Experiments were performed using at least two independent cultures unless noted otherwise. The profiles of organelle divisions and cell cycles were chosen randomly to sample unbiased populations. Division sites are defined as regions with Dnml particle-positive sites or morphologically constricted sites. We used unpaired $t$-test for normal distribution data We used the Mann-Whitney test or Kruskal-Wallis nonparametric ANOVA for skewed distribution of the data. $p$-values in multiple comparison are adjusted with Bonferroni correction. The skewness was determined by Pearson's skewness test in GraphPad Prism 7.4. Confidence levels are shown in each graph.

\section{Data availability}

All relevant data are available from the corresponding author upon reasonable request. The full set of acquired data and results from MS analysis are available in jPOSTrepo (Japan ProteOme STandard Repository, https://repository.jpostdb.org/). The accession numbers are PXD010716 for Proteomechange and JPST000474 for jPOST. A reporting summary for this Article is available as a Supplementary Information file.

Received: 5 April 2018 Accepted: 8 October 2018

Published online: 06 November 2018

\section{References}

1. Danino, D. \& Hinshaw, J. E. Dynamin family of mechanoenzymes. Curr. Opin. Cell Biol. 13, 454-460 (2001). 
2. Hinshaw, J. E. \& Schmid, S. L. Dynamin self-assembles into rings suggesting a mechanism for coated vesicle budding. Nature 374, 690 (1995).

3. Bleazard, W. et al. The dynamin-related GTPase Dnm1 regulates mitochondrial fission in yeast. Nat. Cell Biol. 1, 298-304 (1999).

4. Koch, A. et al. Dynamin-like protein 1 is involved in peroxisomal fission. $J$. Biol. Chem. 278, 8597-8605 (2003).

5. Damke, H., Baba, T., Warnock, D. E. \& Schmid, S. L. Induction of mutant dynamin specifically blocks endocytic coated vesicle formation. J. Cell Biol. 127, 915-934 (1994).

6. Stowell, M. H., Marks, B., Wigge, P. \& McMahon, H. T. Nucleotide-dependent conformational changes in dynamin: evidence for a mechanochemical molecular spring. Nat. Cell Biol. 1, 27-32 (1999).

7. Mears, J. A. et al. Conformational changes in Dnm1 support a contractile mechanism for mitochondrial fission. Nat. Struct. Mol. Biol. 18, 20-26 (2011).

8. Ingerman, E. et al. Dnm1 forms spirals that are structurally tailored to fit mitochondria. J. Cell Biol. 170, 1021-1027 (2005).

9. Warnock, D. E., Hinshaw, J. E. \& Schmid, S. L. Dynamin self-assembly stimulates its GTPase activity. J. Biol. Chem. 271, 22310-22314 (1996).

10. Bourne, H. R., Sanders, D. A. \& McCormick, F. The GTPase superfamily: a conserved switch for diverse cell functions. Nature 348, 125-132 (1990).

11. Koirala, $\mathrm{S}$. et al. Interchangeable adaptors regulate mitochondrial dynamin assembly for membrane scission. Proc. Natl Acad. Sci. USA 110, E1342-E1351 (2013).

12. Kuroiwa, T. et al. Vesicle, mitochondrial, and plastid division machineries with emphasis on dynamin and electron-dense rings. Int. Rev. Cell. Mol. Biol. 271, 97-152 (2008)

13. Suzuki, K. et al. Behavior of mitochondria, chloroplasts and their nuclei during the mitotic cycle in the ultramicroalga Cyanidioschyzon merolae. Eur. J. Cell Biol. 63, 280-288 (1994).

14. Matsuzaki, M. et al. Genome sequence of the ultrasmall unicellular red alga Cyanidioschyzon merolae 10D. Nature 428, 653-657 (2004).

15. Yoshida, Y. et al. The bacterial ZapA-like protein ZED is required for mitochondrial division. Curr. Biol. 19, 1491-1497 (2009).

16. Yoshida, Y. et al. Glycosyltransferase MDR1 assembles a dividing ring for mitochondrial proliferation comprising polyglucan nanofilaments. Proc. Natl Acad. Sci. USA 114, 13284-13289 (2017).

17. Imoto, Y. et al. Single-membrane-bounded peroxisome division revealed by isolation of dynamin-based machinery. Proc. Natl Acad. Sci. USA 110, 9583-9588 (2013)

18. Kuroiwa, T., Suzuki, K. \& Kuroiwa, H. Mitochondrial division by an electron-dense ring in Cyanidioschyzon merolae. Protoplasma 175, 173-177 (1993).

19. Nishida, K. et al. Dynamic recruitment of dynamin for final mitochondrial severance in a primitive red alga. Proc. Natl Acad. Sci. USA 100, 2146-2151 (2003).

20. Takahara, M. et al. Localization of the mitochondrial FtsZ protein in a dividing mitochondrion. Cytologia 66, 421-425 (2001).

21. Kuroiwa, T. et al. Structure, function and evolution of the mitochondrial division apparatus. Biochim. Biophys. Acta 1763, 510-521 (2006).

22. Purkanti, R. \& Thattai, M. Ancient dynamin segments capture early stages of host-mitochondrial integration. Proc. Natl Acad. Sci. USA 112, 2800-2805 (2015).

23. Yoshida, Y. et al. Chloroplasts divide by contraction of a bundle of nanofilaments consisting of polyglucan. Science 329, 949-953 (2010).

24. Imoto, Y. et al. Identification of peroxisome-dividing ring in Cyanidioschyzon merolae based on organelle partner hypothesis. Cytologia 77, 515-522 (2012).

25. Imoto, Y. et al. Defining the dynamin-based ring organizing center on the peroxisome-dividing machinery isolated from Cyanidioschyzon merolae. J. Cell Sci. 130, 853-867 (2017).

26. Nozaki, $\mathrm{H}$. et al. A $100 \%$-complete sequence reveals unusually simple genomic features in the hot-spring red alga Cyanidioschyzon merolae. BMC Biol. 5, 28 (2007)

27. Kim, Y. I., Park, S., Jeoung, D. I. \& Lee, H. Point mutations affecting the oligomeric structure of Nm23-H1 abrogates its inhibitory activity on colonization and invasion of prostate cancer cells. Biochem. Biophys. Res. Commun. 307, 281-289 (2003).

28. Norman, A. W. et al. Detection of phosphohistidine in nucleoside diphosphokinase isolated from Jerusalem artichoke mitochondria. Biochem. Biophys. Res. Commun. 20, 703-709 (1965).

29. Boissan, M., Uwe, S. \& Marie-Lise, L. The NDPK/NME superfamily: state of the art. Lab. Invest. 98, 164-174 (2018).

30. Boissan, M. et al. Nucleoside diphosphate kinases fuel dynamin superfamily proteins with GTP for membrane remodeling. Science 344, 1510-1515 (2014).

31. Izumiya, H. \& Masayuki, Y. Cloning and functional analysis of the ndk1 gene encoding nucleoside-diphosphate kinase in Schizosaccharomyces pombe. J. Biol. Chem. 270, 27859-27864 (1995).
32. Ishijima, Y. et al. Overexpression of nucleoside diphosphate kinases induces neurite outgrowth and their substitution to inactive forms leads to suppression of nerve growth factor-and dibutyryl cyclic AMP-induced effects in PC12D cells. FEBS Lett. 445, 155-159 (1999).

33. Yoshida, Y., Ogura, Y. \& Hasunuma, K. Interaction of nucleoside diphosphate kinase and catalases for stress and light responses in Neurospora crassa. FEBS Lett. 580, 3282-3286 (2006)

34. Sumiya, N., Fujiwara, T., Era, A. \& Miyagishima, S. Y. Chloroplast division checkpoint in eukaryotic algae. Proc. Natl Acad. Sci. USA 113, E7629-E7638 (2016).

35. Miyagishima, S. Y. et al. A plant-specific dynamin-related protein forms a ring at the chloroplast division site. Plant Cell 15, 655-665 (2003).

36. Bohuszewicz, O. \& Low, H. H. Structure of a mitochondrial fission dynamin in the closed conformation. Nat. Struc. Mol. Biol. 25, 722-731 (2018).

37. Krishnan, K. et al. Nucleoside diphosphate kinase, a source of GTP, is required for dynamin-dependent synaptic vesicle recycling. Neuron 30, 197-210 (2001)

38. Kleineke, J., Düls, C. \& Söling, H. D. Subcellular compartmentation of guanine nucleotides and functional relationships between the adenine and guanine nucleotide systems in isolated hepatocytes. FEBS Lett. 107, 198-202 (1979).

39. Hatakeyama, K., Harada, T. \& Kagamiyama, H. IMP dehydrogenase inhibitors reduce intracellular tetrahydrobiopterin levels through reduction of intracellular GTP levels. Indications of the regulation of GTP cyclohydrolase I activity by restriction of GTP availability in the cells. J. Biol. Chem. 267, 20734-20739 (1992).

40. Suzuki, K. et al. Behavior of mitochondria, chloroplasts and their nuclei during the mitotic cycle in ultramicroalga Cyanidioschyzon merolae. Eur. J. Cell Biol. 63, 280-288 (1994).

41. Nishida, K. et al. WD40 protein Mda1 is purified with Dnm1 and forms a dividing ring for mitochondria before Dnm1 in Cyanidioschyzon merolae. Proc. Natl Acad. Sci. USA 104, 4736-4741 (2007).

42. Nishida, K. et al. Triple immunofluorescent labeling of FtsZ, dynamin, and EF-Tu reveals a loose association between the inner and outer membrane mitochondrial division machinery in the red alga Cyanidioschyzon merolae. J. Histochem. Cytochem. 52, 843-849 (2004).

43. Nishida, K. et al. Cell cycle-regulated, microtubule-independent organelle division in Cyanidioschyzon merolae. Mol. Biol. Cell 16, 2493-2502 (2005).

44. Fujiwara, T., Yoshida, Y. \& Kuroiwa, T. Synchronization of cell nuclear, mitochondrial and chloroplast divisions in the unicellular red alga Cyanidioschyzon merolae. Cytologia 74, 1 (2009).

45. Ohnuma, M. et al. Transient gene suppression in a red alga, Cyanidioschyzon merolae 10D. Protoplasma 236, 107-112 (2009).

46. Matsumoto, M. et al. Large-scale analysis of the human ubiquitin-related proteome. Proteomics 5, 4145-4151 (2005).

\section{Acknowledgements}

This work was supported in part by grants from Japan Society for the Promotion of Science Fellowships (no. 14J04556 to Y.I.), MEXT-Supported Program for the Strategic Research Foundation at Private Universities (no. JWU2014-1018 to T.K.), Core Research for Evolutional Science and Technology (CREST) Program of Japan Science and Technology Agency (JST) (to T.K.), Ministry of Education, Culture, Sports, Science, and Technology of Japan, Grants-in-Aid for Scientific Research (no. 17K00676 to M.O.; no. JP16H04813 to T.K; nos. JP24247038, JP25112518, JP25116717, JP26116007, JP15K14511, and JP15K21743 to Y.F.), and grants from the Takeda Science Foundation (to Y.F.), Naito Foundation (to Y.F.) and Japan Foundation for Applied Enzymology and Novartis Foundation (Japan) for the Promotion of Science (to Y.F.). We thank O. Misumi (Yamaguchi University) and F. Yagisawa (Ryukyu University) for C. merolae 10D, Y. Toh (Kyushi University) for supporting electron microscope, and M. Matsumoto (Human Proteome Research Centre, Kyushu University) for LC-MS/MS proteomic analysis and instructive comments, M. Ishii and K. Shimizu (Kyushu University) for generous support to prepare manuscript, K. Itoh (Johns Hopkins University) for discussion and generous support for the research. We also thank S. R. Doctrow and M Arico for helpful comments.

\section{Author contributions}

Y.I. designed the research; Y.I., M.O., and Y.A. performed the research; Y.I., Y.A., K.O. M.H., H.K., and T.K. contributed new reagents/analytical tools; Y.I. and Y.A. analyzed the data; and Y.I., M.H., K.O., H.K., T.K., and Y.F. wrote the paper

\section{Additional information}

Supplementary Information accompanies this paper at https://doi.org/10.1038/s41467 018-07009-z.

Competing interests: The authors declare no competing interests. 
Reprints and permission information is available online at http://npg.nature.com/ reprintsandpermissions/

Publisher's note: Springer Nature remains neutral with regard to jurisdictional claims in published maps and institutional affiliations.

(c) (1)

Open Access This article is licensed under a Creative Commons Attribution 4.0 International License, which permits use, sharing, adaptation, distribution and reproduction in any medium or format, as long as you give appropriate credit to the original author(s) and the source, provide a link to the Creative Commons license, and indicate if changes were made. The images or other third party material in this article are included in the article's Creative Commons license, unless indicated otherwise in a credit line to the material. If material is not included in the article's Creative Commons license and your intended use is not permitted by statutory regulation or exceeds the permitted use, you will need to obtain permission directly from the copyright holder. To view a copy of this license, visit http://creativecommons.org/ licenses/by/4.0/.

(C) The Author(s) 2018 